\title{
Pacific
}

Journal of

Mathematics

\section{RINGEL-HALL ALGEBRAS AND TWO-PARAMETER QUANTIZED ENVELOPING ALGEBRAS}

\author{
XIN TANG
}




\title{
RINGEL-HALL ALGEBRAS AND TWO-PARAMETER QUANTIZED ENVELOPING ALGEBRAS
}

\author{
XIN TANG
}

Let $\mathfrak{g}$ be a finite-dimensional complex simple Lie algebra and $\Lambda$ be the finitedimensional hereditary algebra associated to $\mathfrak{g}$. Let $U_{r, s}^{+}(\mathfrak{g})$ (respectively $\left.U_{r, s}^{\geq 0}(\mathfrak{g})\right)$ denote the two-parameter quantized enveloping algebra of the positive maximal nilpotent (respectively Borel) Lie subalgebra of $\mathfrak{g}$. We study the two-parameter quantized enveloping algebras $U_{r, s}^{+}(\mathfrak{g})$ and $U_{r, s}^{\geq 0}(\mathfrak{g})$ using the approach of Ringel-Hall algebras. First of all, we show that $U_{r, s}^{+}(\mathfrak{g})$ is isomorphic to a certain two-parameter twisted Ringel-Hall algebra $H_{r, s}(\Lambda)$, which generalizes a result of Reineke. Based on detailed computations in $H_{r, s}(\Lambda)$, we show that $H_{r, s}(\Lambda)$ can be presented as an iterated skew polynomial ring. As an result, we obtain a PBW-basis for $H_{r, s}(\Lambda)$, which can be further used to construct a PBW-basis for the two-parameter quantized enveloping algebra $U_{r, s}(\mathfrak{g})$. We also show that all prime ideals of $U_{r, s}^{+}(\mathfrak{g})$ are completely prime under some mild conditions on the parameters $r, s$. Second, we study the two-parameter extended Ringel-Hall algebra $\overline{H_{r, s}(\Lambda)}$. In particular, we define a Hopf algebra structure on $\overline{H_{r, s}(\Lambda)}$; and we prove that $U_{r, s}^{\geq 0}(\mathfrak{g})$ is isomorphic as a Hopf algebra to the two-parameter extended Ringel-Hall algebra $\overline{H_{r, s}(\Lambda)}$.

\section{Introduction}

The interest in two-parameter quantum groups (or multiparameter quantum groups) arose in the early 1990s. Various definitions (or constructions) of two-parameter quantum groups (or multiparameter quantum groups) have appeared in the vast literature [Artin et al. 1991; Chin and Musson 1996; Dobrev and Parashar 1993; Doi and Takeuchi 1994; Jing 1992; Kulish 1990; Reshetikhin 1990; Sudbery 1990]. In particular, Takeuchi [1990] defined the two-parameter quantum groups associated to the general linear Lie algebras $\mathfrak{g l}_{n}$ and the special linear Lie algebras $\mathfrak{s l}_{n}$. These quantum groups are certain two-parameter deformations of the universal enveloping algebras $U(\mathfrak{g})$ of the Lie algebras $\mathfrak{g}$. Motivated by the connections

MSC2000: 17B37.

Keywords: two-parameter quantized enveloping algebras, two-parameter Ringel-Hall algebras,

skew polynomial rings, completely prime ideals.

Partially supported by Fayetteville State University Faculty Development Research Grant. 
to the study of down-up algebras, Takeuchi's two-parameter quantum groups have been reinvestigated by Benkart and Witherspoon [2004b]. In contrast to Takeuchi's two-parameter quantum groups, the two-parameter quantum groups they defined have the opposite coproduct.

Recently, more research efforts have been focused on finding similar constructions of the two-parameter quantum groups associated to other finite-dimensional complex simple Lie algebras $\mathfrak{g}$ and Kac-Moody algebras, and studying their ringtheoretic properties and representation theory [Bergeron et al. 2006; Benkart et al. 2006; Benkart and Witherspoon 2004a; Hu et al. 2008; Hu and Pei 2008]. For the finite-dimensional simple Lie algebras $\mathfrak{g}, \mathrm{Hu}$ and Pei [2008] formulated a uniform construction of the two-parameter quantum groups $U_{r, s}(\mathfrak{g})$ in terms of Ringel form. All these constructions and their modifications can also be unified by using the methods of Kharchenko [2002; 1999], in which a variety of quantum enveloping algebras were constructed from certain quantification matrices. The two-parameter quantum groups $U_{r, s}(\mathfrak{g})$ are similar to the one-parameter quantum groups in many aspects: They are also Hopf algebras and admit both the triangular decompositions and the Drinfeld double realizations. Indeed, they share a similar representation and structure theory with their one-parameter analogue. However, the two-parameter quantum groups possess a more complicated structure and less symmetry, which makes them more difficult to study.

To effectively study the two-parameter quantum groups $U_{r, s}(\mathfrak{g})$, it is natural to first study their important subalgebras such as $U_{r, s}^{+}(\mathfrak{g})$ and $U_{r, s}^{\geq 0}(\mathfrak{g})$, which can be regarded as the two-parameter quantized enveloping algebras of the nilpotent subalgebras $\mathfrak{n}^{+}$and the Borel subalgebras $\mathfrak{b}^{+}$of $\mathfrak{g}$.

In this paper, we will study these algebras from the viewpoint of two-parameter Ringel-Hall algebras. This approach has played a very important role in the study of one-parameter quantum groups $U_{q}(\mathfrak{g})$ [Green 1995; Lusztig 1993; 1990; Ringel 1990b; 1996; 1993; 1990c; 1990a; Xiao 1997]. It is well known that the quantized enveloping algebras $U_{q}(\mathfrak{g})$ can be realized as the reduced Drinfeld doubles of the extended Ringel-Hall algebras $H_{v}^{*}(\Lambda)$ of a certain finite-dimensional hereditary algebra associated to $\mathfrak{g}$. The one-parameter quantized enveloping algebra $U_{q}(\mathfrak{g})$ is first defined via generators and relations. Thus a first priority in the study of $U_{q}(\mathfrak{g})$ is to provide more information on the Hopf algebra structure of $U_{q}(\mathfrak{g})$ and construct good bases for $U_{q}(\mathfrak{g})$ as an algebra. These can be successfully fulfilled by using the Ringel-Hall algebra realization of $U_{q}(\mathfrak{g})$. Furthermore, this realization contributes significantly to the construction of canonical bases for $U_{q}^{+}(\mathfrak{g})$ via the representation theory of finite-dimensional hereditary algebras [Ringel 1996; Lusztig 1990]. For more details about Ringel-Hall algebras and their applications to the study of oneparameter quantum groups $U_{q}(\mathfrak{g})$, see [Green 1995; Ringel 1990b; 1996] and the references therein. 
Let $\mathfrak{g}$ be a finite-dimensional complex simple Lie algebra of type $A, D, E$ and $\Lambda$ be the finite-dimensional hereditary algebra associated to $\mathfrak{g}$. Indeed, $\Lambda$ is the path algebra of the corresponding Dynkin quiver associated to $\mathfrak{g}$. Reineke [2001], for the purpose of studying the monoid ring arising from generic extensions, defined a twoparameter Ringel-Hall algebra $H_{r, s}(\Lambda)$ of $\Lambda$. He proved that $H_{r, s}(\Lambda)$ is isomorphic to Takeuchi's two-parameter quantization $\bigcup_{r, s}\left(\mathfrak{n}^{+}\right)$, where $\bigcup_{r, s}\left(\mathfrak{n}^{+}\right)=U_{r, s}^{+}(\mathfrak{g})$ is the two-parameter quantized enveloping algebra of the nilpotent subalgebra $\mathfrak{n}^{+}$of the Lie algebra $\mathfrak{g}$.

In this paper, we will generalize Reineke's definition to any finite-dimensional complex simple Lie algebra $\mathfrak{g}$. In the case of nonsimply connected Lie algebras, there are no quivers and path algebras available, so we will take $\Lambda$ to be the corresponding finite-dimensional hereditary tensor algebra associated to the k-species [Dlab and Ringel 1975; 1976]. By Ringel's results [1990c; 1990a], the Hall polynomials exist for the extensions between modules in the category $\Lambda$-mod. Reineke's definition of two-parameter Ringel-Hall algebras depends solely on the existence of Hall polynomials, and it can thus be applied to all finite-dimensional complex Lie algebras. For any finite-dimensional simple Lie algebra $\mathfrak{g}$, we shall prove that the two-parameter quantized enveloping algebra $U_{r, s}^{+}(\mathfrak{g})$ is isomorphic to the two-parameter Ringel-Hall algebra $H_{r, s}(\Lambda)$, where $\Lambda$ is the corresponding finite-dimensional hereditary algebra associated to $\mathfrak{g}$ [Dlab and Ringel 1975; 1976].

Following Ringel [1996], we shall carry out some standard calculations inside the algebra $H_{r, s}(\Lambda)$. As a result, we are able to prove that $H_{r, s}(\Lambda)$ can be presented as an iterated skew polynomial ring. An immediate application is that the skewpolynomial ring presentation of $H_{r, s}(\Lambda)$ will yield a natural PBW-basis for $U_{r, s}^{+}(\mathfrak{g})$ through the previous isomorphism. We further prove that all prime ideals of $U_{r, s}^{+}(\mathfrak{g})$ are completely prime based on some mild conditions on the parameters $r, s$. This result has also been proved in [Benkart et al. 2006] for the case of the Lie algebra $\mathfrak{g}=s l_{n}$ by using results in [Kharchenko 2002].

For the purpose of studying the two-parameter quantized enveloping algebra $U_{r, s}^{\geq 0}(\mathfrak{g})$, we will extend the two-parameter Ringel-Hall algebra $H_{r, s}(\Lambda)$ by adding the torus part to it. Furthermore, we will define a Hopf algebra structure on the extended Ringel-Hall algebra $\overline{H_{r, s}(\Lambda)}$. In particular, we will prove that $U_{r, s}^{\geq 0}(\mathfrak{g})$ is isomorphic to the extended Ringel-Hall algebra $\overline{H_{r, s}(\Lambda)}$ as a Hopf algebra. The result gives the possibility of realizing the two-parameter quantum groups $U_{r, s}(\mathfrak{g})$ as the Drinfeld doubles of two-parameter extended Ringel-Hall algebras associated to certain finite-dimensional hereditary algebras $\Lambda$.

The paper is organized as follows. In Section 1, we recall the definition and some basic results of two-parameter quantum groups. In Section 2, we recall Reineke's construction of two-parameter Ringel-Hall algebras and prove some basic results. In Section 3, we define the extended two-parameter Ringel-Hall algebras and then 
propose a Hopf algebra structure for it. We establish the Hopf algebra isomorphism between the two-parameter quantization $U_{r, s}^{\geq 0}(\mathfrak{g})$ and the extended two-parameter Ringel-Hall algebra.

\section{Definition and basic properties of the two-parameter quantum groups $U_{r, s}(\mathfrak{g})$}

Let $\mathfrak{g}$ be a finite-dimensional complex simple Lie algebra. The two-parameter quantum groups $U_{r, s}(\mathfrak{g})$ associated to $\mathfrak{g}$ have been constructed in the literature [Bergeron et al. 2006; Benkart and Witherspoon 2004b; Takeuchi 1990]. A simpler uniform definition of $U_{r, s}(\mathfrak{g})$ in terms of Ringel form has recently been proposed in [Hu and Pei 2008]; we now recall the definition of $U_{r, s}(\mathfrak{g})$ used there and state some basic properties of them.

Let $C=\left(a_{i j}\right)_{i, j \in I}$ be the Cartan matrix corresponding to the Lie algebra $\mathfrak{g}$. Let $\left\{d_{i} \mid i \in I\right\}$ be a set of relatively prime positive integers such that $d_{i} a_{i j}=d_{j} a_{j i}$ for $i, j \in I$. Let $\mathbb{Q}(r, s)$ be the function field in two variables $r, s$ over the field $\mathbb{Q}$ of all rational numbers. We may also choose complex numbers $r, s \in \mathbb{C}$ so that $r^{2} \neq s^{2}$. Let us write $r_{i}=r^{d_{i}}$ and $s_{i}=s^{d_{i}}$.

Let $\langle\cdot, \cdot\rangle$ be the corresponding bilinear form (so-called Ringel or Euler form) defined on the root lattice $2 \cong \mathbb{Z}^{I}$ associated to $\mathfrak{g}$. More precisely, the bilinear form is defined as follows:

$$
\langle i, j\rangle:=\left\langle\alpha_{i}, \alpha_{j}\right\rangle= \begin{cases}d_{i} a_{i j} & \text { if } i<j \\ d_{i} & \text { if } i=j \\ 0 & \text { if } i>j\end{cases}
$$

Definition 1.1 [Bergeron et al. 2006; Benkart and Witherspoon 2004b; Hu and Pei 2008]. The two-parameter quantum groups $U_{r, s}(\mathfrak{g})$ are the $\mathbb{Q}(r, s)$-algebras generated by the generators $e_{i}, f_{i}, w_{i}^{ \pm 1}, w_{i}^{\prime \pm 1}$ subject to the relations

$$
\begin{array}{rlrl}
w_{i}^{ \pm 1} w_{j}^{ \pm 1} & =w_{j}^{ \pm 1} w_{i}^{ \pm 1}, & w_{i}^{ \pm 1} w_{j}^{ \pm 1} & =w_{j}^{ \pm 1} w_{i}^{ \pm 1}, \\
w_{i}^{ \pm 1} w_{j}^{ \pm 1} & =w_{j}^{ \pm 1} w_{i}^{ \pm 1}, & w_{i}^{ \pm 1} w_{i}^{\mp 1} & =1=w_{i}^{ \pm 1} w_{i}^{\prime \mp 1}, \\
w_{i} e_{j} & =r^{\langle j, i\rangle} s^{-\langle i, j\rangle} e_{j} w_{i}, & w_{i}^{\prime} e_{j} & =r^{-\langle i, j\rangle} s^{\langle j, i\rangle} e_{j} w_{i}^{\prime}, \\
w_{i} f_{j} & =r^{-\langle j, i\rangle} s^{\langle i, j\rangle} f_{j} e_{i}, & w_{i}^{\prime} f_{j} & =r^{\langle i, j\rangle} s^{-\langle j, i\rangle} f_{j} w_{i}^{\prime}, \\
e_{i} f_{j}-f_{j} e_{i} & =\delta_{i, j}\left(w_{i}-w_{i}^{\prime}\right) /\left(r_{i}-s_{i}\right), & & \\
1-a_{i j} & & & \\
\sum_{k=0}(-1)^{k}\left(\begin{array}{c}
1-a_{i j} \\
k
\end{array}\right)_{r_{i} s_{i}^{-1}} c_{i j}^{(k)} e_{i}^{1-a_{i j}-k} e_{j} e_{i}^{k}=0 & \text { for } i \neq j, \\
1-a_{i j} & \\
\sum_{k=0}(-1)^{k}\left(\begin{array}{c}
1-a_{i j} \\
k
\end{array}\right)_{r_{i} s_{i}^{-1}} c_{i j}^{(k)} f_{i}^{k} f_{j} f_{i}^{1-a_{i j}-k}=0 & \text { for } i \neq j,
\end{array}
$$


where $c_{i j}^{(k)}=\left(r_{i} s_{i}^{-1}\right)^{k(k-1) / 2} r^{k\langle j, i\rangle} s^{-k\langle i, j\rangle}$ for $i \neq j$, and for a symbol $v$, we set up the notation

$$
\begin{aligned}
(n)_{v} & =\frac{v^{n}-1}{v-1}, \quad(n)_{v} !=(1)_{v}(2)_{v} \cdots(n)_{v} \\
\left(\begin{array}{l}
n \\
k
\end{array}\right)_{v} & =\frac{(n)_{v} !}{(k)_{v} !(n-k)_{v} !} \quad \text { for } n \geq k \geq 0
\end{aligned}
$$

and $(0)_{v} !=1$.

Remark 1.1. In the sequel, if needed, we may change the base field from the function field $\mathbb{Q}(r, s)$ to the complex number field $\mathbb{C}$ by choosing $r, s \in \mathbb{C}$ in so that $r^{m} s^{n}=1$ implies $n=m=0$, or we may restrict the base ring to the rational number field $\mathbb{Q}$ or the local ring $\mathbb{Q}[r, s]_{(r-1, s-1)}$.

From [Bergeron et al. 2006; Benkart and Witherspoon 2004b; Hu and Pei 2008], we know the algebra $U_{r, s}(\mathfrak{g})$ has a Hopf algebra structure with the corresponding comultiplication, counit and antipode defined as follows:

$$
\begin{aligned}
\Delta\left(w_{i}^{ \pm 1}\right) & =w_{i}^{ \pm 1} \otimes w_{i}^{ \pm 1}, & \Delta\left(w_{i}^{\prime \pm 1}\right) & =w_{i}^{\prime \pm 1} \otimes w_{i}^{\prime \pm 1}, \\
\Delta\left(e_{i}\right) & =e_{i} \otimes 1+w_{i} \otimes e_{i}, & \Delta\left(f_{i}\right) & =1 \otimes f_{i}+f_{i} \otimes w_{i}^{\prime}, \\
\epsilon\left(w_{i}^{ \pm 1}\right) & =\epsilon\left(w_{i}^{\prime \pm 1}\right)=1, & \epsilon\left(e_{i}\right) & =\epsilon\left(f_{i}\right)=0, \\
S\left(w_{i}^{ \pm 1}\right) & =w_{i}^{\mp 1}, & S\left(w_{i}^{\prime \pm 1}\right) & =w_{i}^{\prime \mp 1}, \\
S\left(e_{i}\right) & =-w_{i}^{-1} e_{i}, & S\left(f_{i}\right) & =-f_{i} w_{i}^{\prime-1} .
\end{aligned}
$$

Let $U_{r, s}^{+}(\mathfrak{g})$ and $U_{r, s}^{-}(\mathfrak{g})$ be the subalgebras of $U_{r, s}(\mathfrak{g})$ generated by $e_{i}$ for $i \in I$ and by $f_{i}$ for $i \in I$, respectively. Let $U_{r, s}^{0}(\mathfrak{g})$ be the subalgebra of $U_{r, s}(\mathfrak{g})$ generated by $w_{i}^{ \pm 1}, w_{i}^{\prime \pm 1}$ for $i \in I$. The following result about the triangular decomposition of $U_{r, s}(\mathfrak{g})$ was obtained in the papers above.

Proposition 1.1. $U_{r, s}(\mathfrak{g})$ has the standard triangular decomposition

$$
U_{r, s}(\mathfrak{g}) \cong U_{r, s}^{-}(\mathfrak{g}) \otimes U_{r, s}^{0}(\mathfrak{g}) \otimes U_{r, s}^{+}(\mathfrak{g}) .
$$

Let us denote by $\mathbb{Z}^{I}$ the free abelian group of rank $|I|$ with a basis denoted by $z_{1}, z_{2}, \ldots, z_{|I|}$. Given an element $\boldsymbol{a} \in \mathbb{Z}^{I}$, say $\boldsymbol{a}=\sum a_{i} z_{i}$, we set $|\boldsymbol{a}|=\sum a_{i}$. The algebras $U_{r, s}^{+}(\mathfrak{g})$ and $U_{r, s}^{-}(\mathfrak{g})$ are $\mathbb{Z}^{I}$-graded algebras by assigning to the generator $e_{i}$ and $f_{i}$, respectively, the degree $z_{i}$. Given $\boldsymbol{a} \in \mathbb{Z}^{I}$, we denote by $U_{r, s}^{ \pm}(\mathfrak{g})_{\boldsymbol{a}}$ the set of homogeneous elements of degree $\boldsymbol{a}$ in $U_{r, s}^{ \pm}(\mathfrak{g})$; thus we have the decomposition

$$
U_{r, s}^{+}(\mathfrak{g})=\bigoplus_{\boldsymbol{a}} U_{r, s}^{+}(\mathfrak{g})_{\boldsymbol{a}} \quad \text { and } \quad U_{r, s}^{-}(\mathfrak{g})=\bigoplus_{\boldsymbol{a}} U_{r, s}^{-}(\mathfrak{g})_{\boldsymbol{a}} .
$$

Let $U_{r, s}^{\geq 0}(\mathfrak{g})$ (respectively $\left.U_{r, s}^{\leq 0}(\mathfrak{g})\right)$ be the subalgebra of $U_{r, s}(\mathfrak{g})$ generated by $e_{i}, w_{i}^{ \pm 1}$ (respectively $f_{i}, w_{i}^{\prime \pm 1}$ ), then we have the following result. 
Proposition 1.2 [Bergeron et al. 2006; Benkart and Witherspoon 2004b; Hu and Pei 2008]. The algebra $U_{r, s}(\mathfrak{g})$ can be realized as a Drinfeld double of Hopf subalgebras $U_{r, s}^{\geq 0}(\mathfrak{g})$ and $U_{r, s}^{\leq 0}(\mathfrak{g})$ with respect to the pairing $(\cdot, \cdot)$, that is,

$$
U_{r, s}(\mathfrak{g}) \cong D\left(U_{r, s}^{\geq 0}(\mathfrak{g}), U_{r, s}^{\leq 0}(\mathfrak{g})\right) .
$$

To better understand $U_{r, s}(\mathfrak{g})$, it is natural to further study the subalgebras $U_{r, s}^{+}(\mathfrak{g})$ and $U_{r, s}^{\geq 0}(\mathfrak{g})$. We will address this problem in the forthcoming sections via the approach of Ringel-Hall algebras.

\section{Two-parameter Ringel-Hall algebras $H_{r, s}(\Lambda)$}

In this section, we will first recall Reineke's construction of the two-parameter Ringel-Hall algebra $H_{r, s}(\Lambda)$, where $\Lambda$ denotes the finite-dimensional hereditary algebra associated to a complex simple Lie algebra $\mathfrak{g}$ of type $A, D, E$. Indeed, $\Lambda$ is the path algebra of the corresponding Dynkin quiver associated to $\mathfrak{g}$. Then we will define the corresponding two-parameter Ringel-Hall algebra $H_{r, s}(\Lambda)$ of the finitedimensional hereditary algebra $\Lambda$, which is the corresponding finite-dimensional hereditary algebra associated to any finite-dimensional complex simple Lie algebra $\mathfrak{g}$. We will take $\Lambda$ as the tensor algebras of the associated k-species in the nonsimply connected cases. Note that Reineke's construction is still valid due to the existence of Hall polynomials for $\Lambda$-modules.

To further study the properties of $H_{r, s}(\Lambda)$, we will carry out some calculations similar to ones done in [Ringel 1996]. These will yield a skew polynomial ring presentation of $H_{r, s}(\Lambda)$, which immediately enables us to construct a PBW-basis for $H_{r, s}(\Lambda)$. This PBW-basis will be used to construct a PBW-basis for $U_{r, s}(\mathfrak{g})$. Based on certain mild restrictions on the parameters $r, s$, using the stratification theory of prime ideals developed in [Goodearl and Letzter 2000], we will further prove that all prime ideals of $H_{r, s}(\Lambda)$ are completely prime. Finally, we establish the relationship between the algebra $U_{r, s}^{+}(\mathfrak{g})$ and the two-parameter Ringel-Hall algebra $H_{r, s}(\Lambda)$ by proving that they are isomorphic to each other as algebras. Thus all the results obtained on $H_{r, s}(\Lambda)$ can be transformed to $U_{r, s}^{+}(\mathfrak{g})$ via this algebra isomorphism.

2.1. Preliminaries on $\boldsymbol{k}$-species. In this subsection, for the reader's convenience, we shall recall some basic information about $k$-species. The study of $k$-species is a very important research topic that has generated a vast literature. We shall only briefly mention some results that relate the study of $k$-species to the study of finite-dimensional hereditary algebras and Lie algebras, and cite some relevant references. See [Dlab and Ringel 1975; 1976; Ringel 1976] and the references therein for a detailed account of the structure and representation theory of $k$-species 
and their connections to other subjects. In particular, the following presentation of the material is borrowed from the Dlab and Ringel references.

Gabriel [1972] observed that there is a one-to-one correspondence between the set of indecomposable representations of these graphs ("quivers") with a positive definite quadratic form and the set of positive roots of this quadratic form. Later, Bernšteĭn, Gel'fand and Ponomarev [1973] showed that this result can be proved directly, by using appropriate functors (the BGP reflection functors) to construct all indecomposable representations from the simple ones in the same way that the canonical generators of the Weyl group are used to produce all positive roots from the simple roots. Dlab and Ringel $[1975 ; 1976]$ extended this method. To deal with all Dynkin diagrams (not necessarily those of type $A, D$ or $E$ ), they further considered valued graphs (and therefore "species"). For the valued graphs of Dynkin type, they obtained the same one-to-one correspondence between the set of indecomposable representations and the set of positive roots of the corresponding quadratic form, thus generalizing Gabriel's result for the type $A, D$ or $E$ diagrams. In [1976], they also considered valued graphs with positive semidefinite quadratic form (that is, extended Dynkin diagrams) and described all the indecomposable representations up to homogeneous ones.

A valued graph $\Gamma:=(\Gamma, d)$ consists of a finite set $\Gamma$ (of vertices) together with a set $d$ of nonnegative integers $d_{i j}$ for all $i, j \in \Gamma$ such that $d_{i i}=0$ and there exist positive integers $\left\{\epsilon_{i}\right\}_{i \in \Gamma}$ that satisfy

$$
d_{i j} \epsilon_{j}=d_{j i} \epsilon_{i} \quad \text { for all } i, j \in \Gamma .
$$

A pair $\{i, j\}$ of vertices of $\Gamma$ is called an edge of the graph $\Gamma$ if $d_{i j} \neq 0$. An orientation of $\Omega$ of a valued graph $(\Gamma, d)$ is given by assigning each edge $\{i, j\}$ of $\Gamma$ an order (which is denoted by an arrow $i \rightarrow j$ ). We usually call $(\Gamma, d, \Omega)$ a valued quiver. Given any orientation $\Omega$ and any vertex $i \in \Gamma$, we can define a new orientation $s_{i} \Omega$ of $(\Gamma, d)$ by reversing the direction of the arrows along all edges containing $i$. A vertex $i \in \Gamma$ is called a sink (or source) with respect to the orientation $\Omega$ if $i \leftarrow j$ (or $i \rightarrow j$ ) for all neighbor vertices $j \in \Gamma$ of $i$. An orientation is said to be admissible if there is an ordering $i_{1}, i_{2}, \ldots, i_{n}$ of $\Gamma$ such that each vertex $i_{t}$ is a sink with respect to the orientation $s_{i_{t-1}} \cdots s_{i_{2}} s_{i_{1}} \Omega$ for all $1 \leq t \leq n$; such an ordering is called an admissible ordering for $\Omega$.

For a given valued graph $\Gamma=(\Gamma, d)$, one can associate a symmetrizable Cartan matrix $C=\left(a_{i j}\right)_{i, j \in \Gamma}$ by setting the entries of $C$ as follows:

$$
a_{i i}=2 \quad \text { and } \quad a_{i j}=-d_{i j} \quad \text { for } \quad i \neq j \in \Gamma .
$$

Conversely, for any symmetrizable Cartan matrix $C$, one can associate a valued graph $\Gamma_{C}$ as well. It is easy to see that the mapping from the valued graph $(\Gamma, d)$ to the Cartan matrix $C$ defines a one-to-one correspondence between the set of 
valued graphs and the set of symmetrizable Cartan matrices. This correspondence indicates a close relationship between the study of valued graphs and the study of Lie algebras, as we shall further explain below.

Let $k$ denote a finite field and let $(\Gamma, d, \Omega)$ be a valued graph together with an admissible orientation $\Omega$. Following [Gabriel 1973], by definition, a $k$-species $\mathscr{Y}=(\mathcal{M}, \Omega):=\left(F_{i},{ }_{i} M_{j}\right)$ of type $(\Gamma, d, \Omega)$ (which is also called a realization of the valued graph $(\Gamma, d, \Omega)$ in [Dlab and Ringel 1976]) consists of a family of $\left(F_{i}-F_{j}\right)$-bimodules ${ }_{i} M_{j}$, where the fields $F_{i}$ are finite field extensions of $k$ in an algebraic closure of $k$ such that $\operatorname{dim}_{k} F_{i}=\epsilon_{i}$ and $\operatorname{dim}\left({ }_{i} M_{j}\right)_{F_{j}}=d_{i j}$. Note that $\mathscr{Y}$ is called connected provided the corresponding graph is connected; an oriented cycle of $\mathscr{Y}$ is given by a sequence of vertices $i_{1}, i_{2}, \ldots, i_{k-1}, i_{k}=i_{1}$ such that $i_{j} \rightarrow i_{j+1}$ for all $1 \leq j \leq k-1$. From now on, we shall always assume that $(\Gamma, d, \Omega)$ is connected and contains no oriented cycles.

A representation $\left(V_{i},{ }_{j} \phi_{i}\right)$ of the $k$-species $\mathscr{Y}$ is given by a set of vector spaces $\left(V_{i}\right)_{F_{i}}$ and $F_{j}$-linear mappings $V_{i} \otimes{ }_{i} M_{j} \rightarrow V_{j}$. Such a representation is called finite-dimensional provided all the vector spaces $V_{i}$ are finite-dimensional vector spaces. A homomorphism $\alpha=\left(\alpha_{i}\right):\left(V_{i},{ }_{j} \phi_{i}\right) \rightarrow\left(V_{i}^{\prime},{ }_{j} \phi_{i}^{\prime}\right)$ is given by a set of $F_{i}$-linear mappings $\alpha_{i}: V_{i} \rightarrow V_{i}^{\prime}$ such that $\alpha_{j j} \phi_{i}={ }_{j} \phi_{i}^{\prime}\left(\alpha_{i} \otimes 1\right)$. We shall denote by $\operatorname{rep} \mathscr{S}=L(M, \Omega)$ the category of all finite-dimensional representations of $(M, \Omega)$. It is an abelian category. A $k$-species $\mathscr{Y}$ is said to be of finite representation type if the category rep $\mathscr{S}$ has only finitely many indecomposable objects.

[Dlab and Ringel 1975, Theorem B]. A $k$-species is of finite representation type if and only if its diagram is a finite union of Dynkin diagrams.

Given a $k$-species $\mathscr{Y}$, one denotes by $\mathbb{Q}^{\Gamma}$ the rational vector space of all vectors $\boldsymbol{x}=\left(x_{i}\right)_{i \in \Gamma}$ over the rational number field. There is a quadratic form defined on $\mathbb{Q}^{n}$ where $n=|\Gamma|$ as follows: For any $x \in \mathbb{Q}^{n}$, let

$$
(x, x)=\sum \epsilon_{i} x_{i}^{2}-\sum m_{i j} x_{i} x_{j},
$$

where $\epsilon_{i}=\operatorname{dim}_{k} F_{i}$ and $m_{i j}=\operatorname{dim}_{k}\left({ }_{i} M_{j}\right)$. Given any representation $\left(V_{i},{ }_{j} \phi_{i}\right)$ of the $k$-species $(M, \Omega)$, one can define the dimension vector mapping

$$
\operatorname{dim}: L(M, \Omega) \rightarrow \mathbb{Q}^{\Gamma}
$$

by setting $\operatorname{dim}(V)=\left(x_{i}\right)$, where $x_{i}=\operatorname{dim}\left(V_{i}\right)_{F_{i}}$ for all $i \in \Gamma$. Dlab and Ringel [1975; 1976] proved that the $k$-species $\mathscr{S}$ is of finite representation type if and only if the corresponding quadratic form is positive definite, that is, the underlying graph is a Dynkin diagram. In particular, we shall quote the following two results:

[Dlab and Ringel 1976, Proposition 1.2]. (a) $(\Gamma, d)$ is a Dynkin diagram if and only if its quadratic form is positive definite. 
(b) $(\Gamma, d)$ is an extended Dynkin diagram if and only if its quadratic form is positive semi-definite.

[Dlab and Ringel 1976, Proposition 2.6]. Let $(\mathcal{M}, \Omega)$ be a realization of the valued graph $(\Gamma, d)$.

(a) If $(\Gamma, d)$ is a Dynkin diagram, then the mapping dim provides a one-to-one correspondence between all positive roots of $(\Gamma, d)$ and all indecomposable representations in $L(M, \Omega)$.

(b) If $(\Gamma, d)$ is an extended Dynkin diagram, then the mapping $\operatorname{dim}$ provides a oneto-one correspondence between all positive roots of $(\Gamma, d)$ of nonzero defect and all indecomposable representations in $L(M, \Omega)$ of nonzero defect.

The results on the representations of valued graphs can be translated into the representation theory of finite-dimensional associative algebras over a field, or more generally into that of certain classes of artinian rings [Dlab and Ringel 1976]. For any given artinian ring $R$, one can define a valued graph, we will not discuss the detailed construction here. Conversely, for any given $k$-species $\mathscr{S}$ on a given valued graph $(\Gamma, d)$, one can define its associated tensor algebra $\Lambda=T(\mathscr{Y})$ by

$$
\Lambda=\bigoplus_{t \geq 0} \Lambda^{(t)}
$$

where

$$
\Lambda^{(0)}=\prod_{i \in \Gamma} F_{i}, \quad \Lambda^{(1)}=\prod_{h \in \Omega}{ }_{i} M_{j}, \quad \text { and } \quad \Lambda^{(n)}=\Lambda^{(n-1)} \otimes_{\Lambda^{(0)}} \Lambda^{(1)} \quad \text { for } t \geq 2
$$

with the componentwise addition and the multiplication induced by taking tensor products. Note that for an admissible orientation $\Omega$ of $(\Gamma, d)$, the tensor algebra $\Lambda$ of $(\Gamma, d, \Omega)$ is a finite-dimensional hereditary $k$-algebra. An algebra $R$ is said to be of finite representation type if there are only finitely many indecomposable finite-dimensional $R$-modules. Each finite-dimensional hereditary $k$-algebra of finite representation type can be identified with the tensor algebra of some $k$ species. It is well known [Dlab and Ringel 1975] that the category $\Lambda$-mod of finitedimensional $\Lambda$-modules is equivalent to the category $\operatorname{rep}(\mathscr{S})$ of finite-dimensional representations of the $k$-species $\mathscr{S}$ over the field $k$.

[Dlab and Ringel 1975, Theorem C]. A finite-dimensional k-algebra $R$ is hereditary of finite representation type if and only if $R$ is Morita equivalent to the tensor algebra $T(\mathscr{S})$, where $\mathscr{Y}$ is a $k$-species of finite representation type.

In the rest of this paper, we will not distinguish between these two categories. 
2.2. Two-parameter Ringel-Hall algebra $\boldsymbol{H}_{r, s}(\Lambda)$. As above, let $k$ be a finite field. Let $\Lambda$ denote a finite-dimensional associative hereditary algebra over $k$. Denote by $q=|k|$ the cardinality of the base field $k$, and let $v$ be a number such that $v^{2}=q$. We shall also assume that $\Lambda$ is finitary, that is, the cardinality of the extension group $\operatorname{Ext}^{1}\left(S, S^{\prime}\right)$ is finite for any two simple $\Lambda$-modules $S$ and $S^{\prime}$.

It is well known that this finitary condition is satisfied by the algebra $\Lambda$ as long as $\Lambda$ is a finitely generated $k$-algebra over a finite base field $k$. By $\mathscr{P}$, we will denote the set of all isomorphism classes of finite-dimensional $\Lambda$-modules. We set $\mathscr{P}_{1}=\mathscr{P}-0$, where 0 denotes the subset of $\mathscr{P}$ consisting of the only isomorphism class of the zero $\Lambda$-module. For any $\alpha \in \mathscr{P}$, we choose a module representative $u_{\alpha}$ for the isomorphism class $\alpha$. We denote by $a_{\alpha}$ the order of the automorphism group $\operatorname{Aut}_{\Lambda}\left(u_{\alpha}\right)$ of the $\Lambda$-module $u_{\alpha}$. It is easy to see that the number $a_{\alpha}$ is independent of the choices of the representatives $u_{\alpha}$ for any $\alpha \in \mathscr{P}$.

For any given three representatives $u_{\alpha}, u_{\beta}, u_{\gamma}$ of the elements $\alpha, \beta, \gamma \in \mathscr{P}$ respectively, we denote by $g_{\alpha \beta}^{\gamma}$ the number of submodules $N$ of $u_{\gamma}$ satisfying the conditions $N \cong u_{\beta}$ and $u_{\gamma} / N \cong u_{\alpha}$.

For any two given $\Lambda$-modules $M, N$, let us set

$$
\langle M, N\rangle=\operatorname{dim}_{k} \operatorname{Hom}(M, N)-\operatorname{dim}_{k} \operatorname{Ext}^{1}(M, N) .
$$

Since the algebra $\Lambda$ is hereditary, it is well known that $\langle M, N\rangle$ depends only on the dimension vectors $\operatorname{dim}(M)$ and $\operatorname{dim}(N)$ of the $\Lambda$-modules $M$ and $N$. Thus for any given two elements $\alpha, \beta \in \mathscr{P}$, we can define the notation

$$
\langle\alpha, \beta\rangle=\left\langle u_{\alpha}, u_{\beta}\right\rangle
$$

where $u_{\alpha}$ and $u_{\beta}$ are any chosen representatives of $\alpha$ and $\beta$ respectively. Note that $\langle\cdot, \cdot\rangle$ is a bilinear form that is not necessarily symmetric. However, using $\langle\cdot, \cdot\rangle$, we can also define a symmetric bilinear form $(\cdot, \cdot)$ by setting

$$
(\alpha, \beta)=\langle\alpha, \beta\rangle+\langle\beta, \alpha\rangle .
$$

In the rest of this paper, we will be mostly dealing with the form $\langle\cdot, \cdot\rangle$ instead.

Let $\Lambda$-mod denote the category of all finite-dimensional $\Lambda$-modules. Note that there exists a fine symmetry between elements in the category $\Lambda$-mod:

Theorem 2.1 [Green 1995, first formula]. Assume that $\Lambda$ is hereditary and finitary. Let $\alpha, \beta, \alpha^{\prime}, \beta^{\prime} \in \mathscr{P}$. Then

$a_{\alpha} a_{\beta} a_{\alpha^{\prime}} a_{\beta^{\prime}} \sum_{\lambda \in \mathscr{P}} g_{\alpha, \beta}^{\lambda} g_{\alpha^{\prime} \beta^{\prime}}^{\lambda} a_{\lambda}^{-1}=\sum_{\rho, \sigma, \sigma^{\prime}, \tau \in \mathscr{P}} \frac{\left|\operatorname{Ext}^{1}\left(u_{\rho}, u_{\tau}\right)\right|}{\left|\operatorname{Hom}\left(u_{\rho}, u_{\tau}\right)\right|} g_{\rho \sigma}^{\alpha} g_{\rho \sigma^{\prime}}^{\alpha^{\prime}} g_{\sigma^{\prime} \tau}^{\beta} g_{\sigma \tau}^{\beta^{\prime}} a_{\rho} a_{\sigma} a_{\sigma^{\prime}} a_{\tau^{\prime}}$.

Let $\mathfrak{g}$ be a finite-dimensional complex simple Lie algebra of type $A, D$ or $E$; and let $\Lambda$ be the finite-dimensional hereditary algebra associated to $\mathfrak{g}$. As a twoparameter twist of Ringel-Hall algebra, the two-parameter Ringel-Hall algebra 
$H_{r, s}(\Lambda)$ was first defined by Reineke [2001] for the purpose of studying the monoid ring of generic extensions. We will first recall some details of its construction.

Since $\mathfrak{g}$ is a finite-dimensional complex simple Lie algebra of type $A, D$ or $E$, we can associate a Dynkin quiver $\vec{\Delta}$ to the Lie algebra $\mathfrak{g}$, so that the path algebra $\Lambda:=k \vec{\Delta}$ of the Dynkin quiver $\vec{\Delta}$ is a finite-dimensional hereditary algebra of finite representation type. Reineke [2001] introduced a two-parameter RingelHall algebra, which was used to realize Takeuchi's two-parameter quantization $\mathcal{U}_{r, s}\left(\mathfrak{n}^{+}\right)$, with $\mathfrak{n}^{+}$the maximal nilpotent Lie subalgebra of the Lie algebra $\mathfrak{g}$. To avoid colliding notation, we will denote Reineke's version of the two-parameter Ringel-Hall algebra by $H_{r, s}(\Lambda)$ instead of the original $H(2)$. Reineke proved that the two-parameter Ringel-Hall algebra $H_{r, s}(\Lambda)$ is indeed isomorphic to the twoparameter quantization $\boldsymbol{U}_{r, s}^{+}\left(\mathfrak{n}^{+}\right)$.

It is natural to extend Reineke's construction to finite-dimensional complex simple Lie algebras of other types. This can be done in terms of $k$-species due to the existence of Hall polynomials [Ringel 1990a; 1990c]. We will first write down the details of the formulation of the two-parameter Ringel-Hall algebra $H_{r, s}(\Lambda)$ for any complex simple Lie algebra $\mathfrak{g}$ of other types. During the process, the algebra $\Lambda$ is taken as the tensor algebra of the $k$-species associated to the nonsimply connected simple complex Lie algebra $\mathfrak{g}$. Then we will show that two-parameter Ringel-Hall algebra $H_{r, s}(\Lambda)$ is isomorphic to the two-parameter quantization $U_{r, s}^{+}(\mathfrak{g})$ for any Lie algebra $\mathfrak{g}$, which generalizes Reineke's result of [2001].

From now on, we will always assume that $\mathfrak{g}$ is a finite-dimensional complex simple algebra and let $\Lambda$ be the corresponding hereditary path algebra (or the tensor algebra of the $k$-species for nonsimply connected cases). Note that there exist Hall polynomials $F_{M, N}^{L}(x)$ associated to modules $M, N$ and $L$ in $\Lambda$-mod such that for these $\Lambda$-modules, we have $g_{M, N}^{L}=F_{M, N}^{L}(q)$, where $q$ is the cardinality of the base field $k$. For a detailed account of the existence and calculation of Hall polynomials in $\Lambda$-mod, see [Ringel 1996; 1993].

Recall that $\mathscr{P}$ is the set of isomorphism classes of finite-dimensional $\Lambda$-modules. Let us denote by $H_{r, s}(\Lambda)$ the free $\mathbb{Q}(r, s)$-module generated by the elements of the set $\left\{u_{\alpha} \mid \alpha \in \mathscr{P}\right\}$. In addition, we define a multiplication on the free $\mathbb{Q}(r, s)$-module $H_{r, s}(\Lambda)$ by

$$
u_{\alpha} u_{\beta}=\sum_{\lambda \in \mathscr{P}} s^{-\langle\alpha, \beta\rangle} F_{u_{\alpha} u_{\beta}}^{u_{\lambda}}\left(r s^{-1}\right) u_{\lambda} \quad \text { for any } \alpha, \beta \in \mathscr{P} .
$$

Then it is easy to see that we have the following result:

Proposition 2.1 (see also [Reineke 2001]). If $\Lambda$ is the finite-dimensional corresponding hereditary algebra associated to the Lie algebra $\mathfrak{g}$, then the algebra $H_{r, s}(\Lambda)$ is an associative $\mathbb{Q}(r, s)$-algebra under the multiplication defined above. 
The proof is a straightforward verification and we will omit it.

2.3. Ring theoretical properties of $\boldsymbol{H}_{\boldsymbol{r}, s}(\boldsymbol{\Lambda})$. Now we will investigate some ringtheoretic properties of the two-parameter Ringel-Hall algebra $H_{r, s}(\Lambda)$. We first verify some basic identities for $H_{r, s}(\Lambda)$ following along the lines in [Ringel 1996]. These calculations are the same as those done there, with some slight modifications.

First, we introduce a new $\mathbb{Q}(r, s)$-basis for $H_{r, s}(\Lambda)$. For any chosen element $\alpha \in \mathscr{P}$, we have an element $u_{\alpha} \in H_{r, s}(\Lambda)$. We denote by $\epsilon(\alpha)$ the $k$-dimension of the endomorphism ring of the module representative $u_{\alpha}$ corresponding to $\alpha$.

For any given module $M$ of the algebra $\Lambda$, we denote the isomorphism class of $M$ by $[M]$ and by $\operatorname{dim}(M)$ the dimension vector of $M$, which is an element of the Grothendieck group $K_{0}(\Lambda)$ of the category $\Lambda$-mod of all finite $\Lambda$-modules modulo the exact sequences.

According to [Bernšteĭn et al. 1973; Dlab and Ringel 1975; Gabriel 1972], there is a one-to-one correspondence between the set of all positive roots for the Lie algebra $\mathfrak{g}$ and the set of indecomposable modules in $\Lambda$-mod (see Section 2.1). Let $a \in \Phi^{+}$be any positive root; we denote by $M(\boldsymbol{a})$ the indecomposable module corresponding to $\boldsymbol{a}$. For any map $\alpha: \Phi^{+} \rightarrow \mathbb{N}_{0}$, set

$$
M(\alpha)=M_{\Lambda}(\alpha)=\bigoplus_{\boldsymbol{a} \in \Phi^{+}} \alpha(\boldsymbol{a}) M(\boldsymbol{a}) .
$$

Then it is easy to see there is a bijection between the set $\mathscr{P}$ of isomorphism classes of finite-dimensional $\Lambda$-modules and the set of all maps $\alpha: \Phi^{+} \rightarrow \mathbb{N}_{0}$. We will not distinguish an element $\alpha \in \mathscr{P}$ from the corresponding map associated to $\alpha$, and we may denote both of them by $\alpha$ if no confusion arises.

For any $\alpha \in \mathscr{P}$, let us set $\operatorname{dim} \alpha=\sum_{\boldsymbol{a} \in \Phi^{+}} \alpha(\boldsymbol{a}) \boldsymbol{a}$. Then we have

$$
\operatorname{dim}(M(\alpha))=\operatorname{dim} \alpha .
$$

For any given $\alpha \in \mathscr{P}$, we denote by $\operatorname{dim}(\alpha)=\operatorname{dim}\left(u_{\alpha}\right)$ the dimension of the $\Lambda$-module $u_{\alpha}$ as a $k$-vector space. Furthermore, let us set

$$
\left\langle u_{\alpha}\right\rangle=s^{\operatorname{dim}\left(u_{\alpha}\right)-\epsilon(\alpha)} u_{\alpha} .
$$

For convenience, we may sometimes simply denote $u_{\alpha}$ by $\alpha$ for any $\alpha \in \mathscr{P}$ and denote $F_{u_{\alpha} u_{\beta}}^{u_{\lambda}}\left(r s^{-1}\right)$ by $g_{\alpha \beta}^{\lambda}$ if no confusion arises. In particular, we will carry out all the computations in terms of $\alpha$ instead of $u_{\alpha}$ in the rest of this subsection.

Obviously the set $\{\langle\alpha\rangle \mid \alpha \in \mathscr{P}\}$ is also a $\mathbb{Q}(r, s)$-basis for the algebra $H_{r, s}(\Lambda)$. Note that $\left\langle\alpha_{i}\right\rangle=\alpha_{i}$ for any element $\alpha_{i} \in \mathscr{P}$ corresponding to the simple root $\alpha_{i}$. Thus the multiplication in $H_{r, s}(\Lambda)$ can be rewritten in terms of this new basis as

$$
\langle\alpha\rangle\langle\beta\rangle=s^{-\epsilon(\alpha)-\epsilon(\beta)-\langle\operatorname{dim} \alpha, \operatorname{dim} \beta\rangle} \sum_{\lambda \in \mathscr{P}} s^{\epsilon(\lambda)} g_{\alpha \beta}^{\lambda}\langle\lambda\rangle \quad \text { for any } \alpha, \beta \in \mathscr{P} .
$$


Furthermore, let us write $e(\alpha, \beta)=\operatorname{dim}_{k} \operatorname{Hom}_{\Lambda}(M(\alpha), M(\beta)) \quad$ and $\quad \zeta(\alpha, \beta)=\operatorname{dim}_{k} \operatorname{Ext}_{\Lambda}^{1}(M(\alpha), M(\beta))$.

Then we have the following proposition, similar to the one in [Ringel 1996]. Proposition 2.2. Let $\alpha_{1}, \ldots, \alpha_{t} \in \mathscr{P}$ such that for $i<j$, we have both $\epsilon\left(\alpha_{j}, \alpha_{i}\right)=0$ and $\zeta\left(\alpha_{i}, \alpha_{j}\right)=0$. Then

$$
\left\langle\bigoplus_{i=1}^{t} \alpha_{i}\right\rangle=\left\langle\alpha_{1}\right\rangle \cdots\left\langle\alpha_{t}\right\rangle
$$

Proof. Without of loss of generality, we may assume that $t=2$. Let us set $\alpha_{1}=\alpha$ and $\alpha_{2}=\beta$. Since $\zeta(\alpha, \beta)=0$, we have

$$
\langle\alpha, \beta\rangle=e(\alpha, \beta)-\zeta(\alpha, \beta)=e(\alpha, \beta) .
$$

Since $e(\beta, \alpha)=0$, we also have

$$
\begin{aligned}
e(\alpha \oplus \beta) & =e(\alpha, \alpha)+e(\alpha, \beta)+e(\beta, \beta)+e(\beta, \alpha) \\
& =e(\alpha, \alpha)+e(\beta, \beta)+e(\alpha, \beta) .
\end{aligned}
$$

Thus

$\epsilon(\alpha \oplus \beta)-\langle\alpha, \beta\rangle-e(\alpha, \alpha)-e(\beta, \beta)$

$$
=e(\alpha, \alpha)+e(\beta, \beta)+e(\alpha, \beta)-e(\alpha, \beta)+\zeta(\alpha, \beta)-e(\alpha, \alpha)-e(\beta, \beta)=0 .
$$

Since $\zeta(\alpha, \beta)=0$, that $g_{\alpha \beta}^{\gamma} \neq 0$ implies that $\gamma=\alpha \oplus \beta$. Since $e(\beta, \alpha)=0$, we have $g_{\alpha \beta}^{\alpha \oplus \beta}=1$. Therefore, we may finish the proof:

$$
\begin{aligned}
\langle\alpha\rangle\langle\beta\rangle & =s^{\operatorname{dim}(\alpha)+\operatorname{dim}(\beta)-\epsilon(\alpha)-\epsilon(\beta)} \alpha \beta \\
& =s^{\epsilon(\alpha \oplus \beta)-\langle\alpha, \beta\rangle-\epsilon(\alpha)-\epsilon(\beta)} g_{\alpha \beta}^{\alpha \oplus \beta}\langle\alpha \oplus \beta\rangle \\
& =\langle\alpha \oplus \beta\rangle .
\end{aligned}
$$

Theorem 2.2. Let $\alpha, \beta \in \mathscr{P}$ such that $e(\beta, \alpha)=0$ and $\zeta(\alpha, \beta)=0$. Then we have

$$
\langle\beta\rangle\langle\alpha\rangle=r^{\langle\alpha, \beta\rangle} s^{-\langle\beta, \alpha\rangle}\langle\alpha\rangle\langle\beta\rangle+\sum_{\gamma \in J(\alpha, \beta)} c_{\gamma}\langle\gamma\rangle
$$

where the coefficients $c_{\gamma}$ are in $\mathbb{Z}\left[r^{ \pm 1}, s^{ \pm 1}\right]$ and $J(\alpha, \beta)$ is the set of all elements $\lambda \in \mathscr{P}$ that are different from $\alpha \oplus \beta$ and $g_{\alpha \beta}^{\lambda} \neq 0$.

Proof. First, by Proposition 2.2, we have $\langle\alpha\rangle\langle\beta\rangle=\langle\alpha \oplus \beta\rangle$.

Note that $\langle\beta\rangle\langle\alpha\rangle=\sum_{\gamma} c_{\gamma}^{\prime} \gamma$. Thus we have the relationship $c_{\gamma}^{\prime}=s^{\operatorname{dim}(\gamma)-\epsilon(\gamma)} c_{\gamma}$ between the coefficients $c_{\gamma}$ and $c_{\gamma}^{\prime}$. By [Ringel 1996], we also have

$$
g_{\beta \alpha}^{\alpha \oplus \beta}=\left(r s^{-1}\right)^{e(\alpha, \beta)} .
$$


Note that $\epsilon(\alpha \oplus \beta)=\epsilon(\alpha)+\epsilon(\beta)+e(\alpha, \beta)$. Thus

$$
\begin{aligned}
c_{\alpha \oplus \beta}^{\prime} & =s^{\operatorname{dim}(\beta)-\epsilon(\beta)+\operatorname{dim}(\alpha)-\epsilon(\alpha)} s^{-\langle\beta, \alpha\rangle} g_{\beta \alpha}^{\alpha \oplus \beta} \\
& =s^{\operatorname{dim}(\alpha \oplus \beta)-\epsilon(\alpha)-\epsilon(\beta)+\zeta(\beta, \alpha)}\left(r s^{-1}\right)^{e(\alpha, \beta)} \\
& =s^{\operatorname{dim}(\alpha \oplus \beta)-\epsilon(\alpha)-\epsilon(\beta)+\zeta(\beta, \alpha)-e(\alpha, \beta)} r^{e(\alpha, \beta)} \\
& =r^{e(\alpha, \beta)} s^{\zeta(\beta, \alpha)} s^{\operatorname{dim}(\alpha \oplus \beta)-\epsilon(\alpha \oplus \beta)}=r^{\langle\alpha, \beta\rangle} s^{-\langle\beta, \alpha\rangle} s^{\operatorname{dim}(\alpha \oplus \beta)-\epsilon(\alpha \oplus \beta)} .
\end{aligned}
$$

Finally, we have $c_{\alpha \oplus \beta}^{\prime} \alpha \oplus \beta=r^{\langle\alpha, \beta\rangle} s^{-\langle\beta, \alpha\rangle}\langle\alpha \oplus \beta\rangle$.

According to the representation theory of finite-dimensional hereditary algebras of finite representation type [Bernšteľn et al. 1973; Dlab and Ringel 1976], we can give a total ordering on the set of positive roots of the Lie algebra $\mathfrak{g}$. Following Ringel [1996], we will order all positive roots in a way $\boldsymbol{a}_{1}, \boldsymbol{a}_{2}, \ldots, \boldsymbol{a}_{m}$ so that $\operatorname{Hom}\left(M\left(\boldsymbol{a}_{i}\right), M\left(\boldsymbol{a}_{j}\right)\right) \neq 0$ implies $i \leq j$, where $M\left(\boldsymbol{a}_{i}\right)$ is the indecomposable module corresponding to the positive root $\boldsymbol{a}_{i}$. Such an ordering will be called $\vec{\Delta}$-admissible.

Lemma 2.1 [Ringel 1996]. A total ordering $\boldsymbol{a}_{1}, \ldots, \boldsymbol{a}_{m}$ of all the positive roots is $\vec{\Delta}$-admissible if and only if $\left\langle\boldsymbol{a}_{i}, \boldsymbol{a}_{j}\right\rangle>0$ implies $i \leq j$. Such an ordering has the additional property that $\left\langle\boldsymbol{a}_{i}, \boldsymbol{a}_{j}\right\rangle<0$ implies $i>j$.

From now on, we will always fix such a $\vec{\Delta}$-admissible ordering on the set of all positive roots.

Proposition 2.3. For any $\alpha \in \mathscr{P}$, we have $\langle\alpha\rangle=\left\langle\alpha\left(\boldsymbol{a}_{1}\right) \boldsymbol{a}_{1}\right\rangle \cdots\left\langle\alpha\left(\boldsymbol{a}_{m}\right) \boldsymbol{a}_{m}\right\rangle$.

Proof. Since the ordering of the positive roots $\boldsymbol{a}_{i}$ is admissible, $e\left(\boldsymbol{a}_{j}, \boldsymbol{a}_{i}\right)=0$ for any $i<j$. In addition, we also have $\zeta\left(\boldsymbol{a}_{i}, \boldsymbol{a}_{j}\right)=0$. Note that, as a module, $\alpha=\bigoplus_{i=1}^{m} \alpha\left(\boldsymbol{a}_{i}\right) M\left(\boldsymbol{a}_{i}\right)$; then the result follows from Proposition 2.2.

Now let us consider the divided powers of $\langle\boldsymbol{a}\rangle$ by setting

$$
\langle\boldsymbol{a}\rangle^{(t)}=\frac{1}{[t]_{\epsilon(\boldsymbol{a})}^{!}}\langle\boldsymbol{a}\rangle^{t}, \quad \text { where }[t]_{\epsilon(\boldsymbol{a})}^{!}=\prod_{i=1}^{t} \frac{r^{i \epsilon(\boldsymbol{a})}-s^{i \epsilon(\boldsymbol{a})}}{r^{\epsilon(\boldsymbol{a})}-s^{\epsilon(a)}} .
$$

Lemma 2.2. Let $\boldsymbol{a}$ be a positive root and $t \geq 0$ be an integer. Then $\langle t \boldsymbol{a}\rangle=\langle\boldsymbol{a}\rangle^{(t)}$.

Proof. The proof is adapted from [Ringel 1996]. Let $\mathscr{S}$ be a reduced $k$-species, where $k$ is a finite field. Then the number of filtrations

$$
t M_{\varphi}(\boldsymbol{a})=M_{0} \supset M_{1} \cdots \supset M_{t}=0
$$

of the module $t M_{S}(\boldsymbol{a})$ with composition factors isomorphic to the module $M_{\mathscr{S}}(\boldsymbol{a})$ is given by evaluating the following polynomial in $x$ at the number $|k|=r s^{-1}$ :

$$
\frac{\left.\left(x^{\epsilon(\boldsymbol{a}) t}-1\right)\left(x^{\epsilon(\boldsymbol{a})(t-1)}-1\right) \cdots\left(x^{\epsilon(\boldsymbol{a})}-1\right)\right)}{\left.\left(x^{\epsilon(\boldsymbol{a})}\right)-1\right)^{t}} .
$$


Since $\zeta(\boldsymbol{a}, \boldsymbol{a})=0$, we have

$$
\boldsymbol{a}^{t}=s^{-\epsilon(\boldsymbol{a})\left(\begin{array}{l}
t \\
2
\end{array}\right)}[t] !_{\epsilon(\boldsymbol{a})} s^{-\epsilon(\boldsymbol{a})\left(\begin{array}{l}
t \\
2
\end{array}\right)} t \boldsymbol{a}=s^{-\epsilon(\boldsymbol{a}) t(t-1)}[t] !_{\epsilon(\boldsymbol{a})} t \boldsymbol{a} .
$$

Therefore,

$$
\begin{aligned}
\langle\boldsymbol{a}\rangle^{(t)} & =\frac{1}{[t] !_{\epsilon(\boldsymbol{a})}}\langle\boldsymbol{a}\rangle^{t}=\frac{s^{-\epsilon(\boldsymbol{a}) t(t-1)}[t] !_{\epsilon(\boldsymbol{a})} s^{t(\operatorname{dim}(\boldsymbol{a})-\epsilon(\boldsymbol{a}))}}{[t] !_{\epsilon(\boldsymbol{a})}} \boldsymbol{a}^{t} \\
& =s^{-\epsilon(\boldsymbol{a}) t(t-1)+t(\operatorname{dim}(\boldsymbol{a})-\epsilon(\boldsymbol{a}))} t \boldsymbol{a} \\
& =s^{t \operatorname{dim}(\boldsymbol{a})-\epsilon(\boldsymbol{a}) t(t-1+1)} t \boldsymbol{a}=s^{t \operatorname{dim}(\boldsymbol{a})-\epsilon(\boldsymbol{a}) t^{2}}=s^{t \operatorname{dim}(\boldsymbol{a})-\epsilon(t \boldsymbol{a})} t \boldsymbol{a}=\langle t \boldsymbol{a}\rangle .
\end{aligned}
$$

For each positive root $\boldsymbol{a}_{i}$, let us define the symbol $X_{i}=\left\langle\boldsymbol{a}_{i}\right\rangle$.

Proposition 2.4. Let $\alpha \in \mathscr{P}$. Then $\alpha$ can be regarded as a map $\alpha: \Phi^{+} \rightarrow \mathbb{N}_{0}$. Setting $\alpha(i)=\alpha\left(\boldsymbol{a}_{i}\right)$, we have

$$
\langle\alpha\rangle=X_{1}^{(\alpha(1))} \cdots X_{m}^{(\alpha(m))}=\left(\prod_{i=1}^{m} \frac{1}{[\alpha(i)]_{\epsilon\left(a_{i}\right)}^{!}}\right) X_{1}^{\alpha(1)} \cdots X_{m}^{\alpha(m)} .
$$

Proof. By Proposition 2.3, we have $\langle\alpha\rangle=\left\langle\alpha(1) \boldsymbol{a}_{1}\right\rangle \cdots\left\langle\alpha(m) \boldsymbol{a}_{m}\right\rangle$. By Lemma 2.2, we also have $\left\langle\alpha(i) \boldsymbol{a}_{i}\right\rangle=X_{i}^{(\alpha(i))}$. Thus the first equality holds. The second equality can be proved by using the divided powers.

Theorem 2.3. The monomials $X_{1}^{\alpha(1)} \ldots X_{m}^{\alpha(m)}$ with $\alpha(1), \ldots, \alpha(m) \in \mathbb{N}_{0}$ form a $\mathbb{Q}(r, s)$-basis of $H_{r, s}(\Lambda)$, and for $i<j$, we have $X_{j} X_{i}=r^{\left\langle\operatorname{dim} X_{i}, \operatorname{dim} X_{j}\right\rangle} s^{-\left\langle\operatorname{dim} X_{j}, \operatorname{dim} X_{i}\right\rangle} X_{i} X_{j}$

$$
+\sum_{I(i, j)} c\left(a_{i+1}, \ldots, a_{j-1}\right) X_{i+1}^{a_{i+1}} \cdots X_{j-1}^{a_{j-1}}
$$

with coefficients $c\left(a_{i+1}, \ldots, a_{j-1}\right)$ in $\mathbb{Q}(r, s)$. Here the index set $I(i, j)$ is the set of sequences $\left(a_{i+1}, \cdots a_{j-1}\right)$ of natural numbers such that $\sum_{t=i+1}^{j-1} a_{t} \boldsymbol{a}_{\boldsymbol{t}}=\boldsymbol{a}_{\boldsymbol{i}}+\boldsymbol{a}_{\boldsymbol{j}}$.

Proof. Given $\alpha(1), \ldots, \alpha(m) \in \mathbb{N}_{0}$, define an element $\alpha \in \mathscr{P}$ by setting $\alpha\left(\boldsymbol{a}_{i}\right)=\alpha(i)$. According to the previous proposition, we have $\langle\alpha\rangle=X_{1}^{(\alpha(1))} \cdots X_{m}^{(\alpha(m))}$. Thus the monomials given in the theorem are exactly nonzero scalar multiples of the elements in $\mathscr{P}$. Therefore, these monomials form a $\mathbb{Q}(r, s)$-basis of $H_{r, s}(\Lambda)$.

Let $i<j$. We can apply Theorem 2.2 to the positive roots $\boldsymbol{a}_{i}$ and $\boldsymbol{a}_{j}$. We need to show that for any $\beta \in J(i, j)$, the element $\beta$ is a scalar multiple of some monomials $X_{i+1}^{a_{i+1}} \cdots X_{j}^{a_{j}}$ with $\sum_{t=i+1}^{j-1} a_{t} \boldsymbol{a}_{t}=\boldsymbol{a}_{i}+\boldsymbol{a}_{j}$.

Let $\beta \in J(i, j)$, and let $\beta(t)=\beta\left(\boldsymbol{a}_{t}\right)$. Since $g_{\boldsymbol{a}_{j} \boldsymbol{a}_{i}}^{\beta} \neq 0$, there is an exact sequence

$$
0 \rightarrow M\left(\boldsymbol{a}_{i}\right) \rightarrow \bigoplus_{t=1}^{m} \beta(t) M\left(\boldsymbol{a}_{t}\right) \rightarrow M\left(\boldsymbol{a}_{j}\right) \rightarrow 0 .
$$


Let us write $f=\left(f_{t}\right)_{t}$ with $f_{t}: M\left(\boldsymbol{a}_{i}\right) \rightarrow \beta(t) M\left(\boldsymbol{a}_{t}\right)$. The exact sequence does not split; otherwise, $\beta=\boldsymbol{a}_{i} \oplus \boldsymbol{a}_{j}$, which contradicts the assumption that $\beta \in J(i, j)$. Let us consider some $t$ with $\beta(t)>0$. We claim that $f_{t} \neq 0$. Otherwise, the cokernel of $f$ would split off $\beta(t)$ copies of $M\left(a_{t}\right)$; and since the cokernel of $f$ is indecomposable, this would mean that the exact sequence splits. Since $\operatorname{Hom}\left(\boldsymbol{a}_{i}, \boldsymbol{a}_{j}\right) \neq 0$, it follows that $i \leq t$. In addition, we can exclude the case $i=t$, since in this case, $f_{t}$ and therefore $f$ would be a split monomorphism. Altogether, we have $i<t$. The dual argument applied to $g$ shows that $t<j$. According to Proposition 2.2, we know that $\langle\beta\rangle$ is a scalar multiple of $X_{i+1}^{a_{i+1}} \cdots X_{j}^{a_{j}}$. The exact sequence exhibited above shows that $\sum_{t=i+1}^{j-1} a_{t} \boldsymbol{a}_{t}=\boldsymbol{a}_{i}+\boldsymbol{a}_{j}$.

Now we define some algebra automorphisms and skew derivations on $H_{r, s}(\Lambda)$. Namely, for any $d \in \mathbb{Z}^{n}$, there is an algebra automorphism $l_{d}$ of $H_{r, s}(\Lambda)$ defined by $l_{d}(w)=r^{\langle\operatorname{dim} w, d\rangle} s^{-\langle d, \operatorname{dim} w\rangle} w$, where $w$ is any homogeneous element of $H_{r, s}(\Lambda)$.

Lemma 2.3 [Ringel 1996]. Let $R$ be a ring and let $l$ be an endomorphism of $R$. For any $r \in R$, we define a map $\delta_{r}: R \rightarrow R$ by

$$
\delta_{r}(x)=r x-l(x) r \quad \text { for any } x \in R .
$$

Then the map $\delta_{r}$ is an l-derivation.

Prooffrom [Ringel 1996]. First, the map $\delta_{r}$ is additive. In addition, for any $x, y \in R$, we have

$$
\begin{aligned}
\delta_{r}(x y)=r x y-l(x y) r & =r x y-l(x) r y+l(x) r y-l(x) l(y) r \\
& =(r x-l(x) r) y+l(x)(r y-l(y) r) \\
& =\delta_{r}(x) y+l(x) \delta_{r}(y) .
\end{aligned}
$$

Thus the map $\delta_{r}$ is an $l$-derivation of $R$.

Definition 2.1. Let $R$ be a domain with $1 \neq 0$, let $\sigma_{1}: R \rightarrow R$ be a ring homomorphism and let $\delta_{1}: R \rightarrow R$ be a $\sigma_{1}$-derivation, so that for all $a, b \in R$, we have

- $\sigma_{1}(a+b)=\sigma_{1}(a)+\sigma_{1}(b)$,

- $\sigma_{1}(a b)=\sigma_{1}(a) \sigma_{1}(b)$,

- $\delta_{1}(a+b)=\delta_{1}(a)+\delta_{1}(b)$,

- $\delta_{1}(a b)=\delta_{1}(a) b+\sigma_{1}(a) \delta_{1}(b)$.

Then the skew polynomial ring $R\left[X_{1}, \sigma_{1}, \delta_{1}\right]$ is the set of noncommutative polynomials $R\left[X_{1}\right]$ with addition defined as commutative polynomials, and with multiplication defined distributively over addition and by the commutator rule

$$
X_{1} a=\sigma_{1}(a) X_{1}+\delta_{1}(a),
$$


valid for all $a \in R$. We set $R_{1}=R\left[X_{1}, \sigma_{1}, \delta_{1}\right]$, and let $\sigma_{2}$ be a ring homomorphism of $R_{1}$ and $\delta_{2}$ be a $\sigma_{2}$-derivation of the ring $R_{1}$. Then we can define another skew polynomial ring $R_{2}=R_{1}\left[X_{2}, \sigma_{2}, \delta_{2}\right]$. Similarly, we can iterate this process to define $R_{n}$ for any $n \geq 2$. These rings $R_{n}$ are called iterated skew polynomial rings.

Let $H_{j}$ denote the $\mathbb{Q}(r, s)$-subalgebra of $H_{r, s}(\Lambda)$ generated by the generators $X_{1}, \ldots, X_{j}$. Thus we have $H_{0}=\mathbb{Q}(r, s)$ and for any $0 \leq j \leq m$, we have

$$
H_{j}=H_{j-1}\left[X_{j}, l_{j}, \delta_{j}\right]
$$

with the automorphism $l_{j}$ and the $l_{j}$-derivation $\delta_{j}$ of $H_{j-1}$. The automorphism $l_{j}$ can be explicitly defined by

$$
l_{j}\left(X_{i}\right)=r^{\left\langle\operatorname{dim} X_{i}, \operatorname{dim} X_{j}\right\rangle} s^{-\left\langle\operatorname{dim} X_{j}, \operatorname{dim} X_{i}\right\rangle} X_{i} \quad \text { for } i<j .
$$

The skew derivation $\delta_{j}$ can be defined by

$$
\delta_{j}\left(X_{i}\right)=X_{j} X_{i}-l_{j}\left(X_{i}\right) X_{j}=\sum_{I(i, j)} c\left(a_{i+1}, \ldots, a_{j-1}\right) X_{i+1}^{a_{i+1}} \cdots X_{j_{1}}^{a_{j-1}} .
$$

Theorem 2.4. The automorphism $l_{j}$ and the skew derivation $\delta_{j}$ satisfy the relation

$$
l_{j} \delta_{j}=r^{\left\langle\boldsymbol{a}_{j}, \boldsymbol{a}_{j}\right\rangle} s^{-\left\langle\boldsymbol{a}_{j}, \boldsymbol{a}_{j}\right\rangle} \delta_{j} l_{j} .
$$

Proof. Suppose $i<j$. Then $l_{j}\left(X_{i}\right)=r^{\left\langle\boldsymbol{a}_{i}, \boldsymbol{a}_{j}\right\rangle} s^{-\left\langle\boldsymbol{a}_{j}, \boldsymbol{a}_{i}\right\rangle} X_{i}$. Thus, we have

$$
\delta_{j} l_{j}\left(X_{i}\right)=r^{\left\langle a_{i}, a_{j}\right\rangle} s^{-\left\langle a_{j}, a_{i}\right\rangle} \delta_{j}\left(X_{i}\right) .
$$

Let us write $\boldsymbol{d}=\boldsymbol{a}_{i}+\boldsymbol{a}_{j}$. Note that $\delta_{j}\left(X_{i}\right)$ is a linear combination of monomials of the form

$$
X_{i+1}^{a_{i+1}} \cdots X_{j-1}^{a_{j-1}} \quad \text { where } \sum_{t=i+1}^{j-1} a_{t} \boldsymbol{a}_{t}=\boldsymbol{a}_{i}+\boldsymbol{a}_{j}=\boldsymbol{d} .
$$

Thus we know that $\delta_{j}\left(X_{i}\right)$ belongs to $H_{r, s}(\Lambda)$. Since we have

$$
\begin{aligned}
& \left\langle\boldsymbol{d}, \boldsymbol{a}_{j}\right\rangle=\left\langle\boldsymbol{a}_{i}+\boldsymbol{a}_{j}, \boldsymbol{a}_{j}\right\rangle=\left\langle\boldsymbol{a}_{i}, \boldsymbol{a}_{j}\right\rangle+\left\langle\boldsymbol{a}_{j}, \boldsymbol{a}_{j}\right\rangle, \\
& \left\langle\boldsymbol{a}_{j}, \boldsymbol{d}\right\rangle=\left\langle\boldsymbol{a}_{j}, \boldsymbol{a}_{i}+\boldsymbol{a}_{j}\right\rangle=\left\langle\boldsymbol{a}_{j}, \boldsymbol{a}_{i}\right\rangle+\left\langle\boldsymbol{a}_{j}, \boldsymbol{a}_{j}\right\rangle,
\end{aligned}
$$

it follows that

$$
\begin{aligned}
l_{j} \delta_{j}\left(X_{i}\right) & =r^{\left\langle\boldsymbol{d}, \boldsymbol{a}_{j}\right\rangle} s^{-\left\langle\boldsymbol{d}, \boldsymbol{a}_{j}\right\rangle} \delta_{j}\left(X_{i}\right) \\
& =r^{\left\langle\boldsymbol{a}_{i}, \boldsymbol{a}_{j}\right\rangle+\left\langle\boldsymbol{a}_{j}, \boldsymbol{a}_{j}\right\rangle} s^{-\left\langle\boldsymbol{a}_{j}, \boldsymbol{a}_{j}\right\rangle-\left\langle a_{j}, \boldsymbol{a}_{j}\right\rangle} \delta_{j}\left(X_{i}\right) \\
& =r^{\left\langle\boldsymbol{a}_{j}, \boldsymbol{a}_{j}\right\rangle} s^{-\left\langle\boldsymbol{a}_{j}, \boldsymbol{a}_{j}\right\rangle} \delta_{j} l_{j}\left(X_{i}\right) .
\end{aligned}
$$

Theorem 2.5. The two-parameter Ringel-Hall algebra $H_{r, s}(\Lambda)$ can be presented as an iterated skew polynomial ring.

Proof. The proof follows from the previous theorem. 
Let $R$ be a ring. Recall that an ideal $P \subset R$ is said to be prime if $P \neq R$ and if whenever the product $A B$ of two ideals $A$ and $B$ of $R$ is contained in $P$, at least one of $A$ and $B$ is contained in $P$. An ideal $P \subset R$ is called completely prime if $P \neq R$ and if whenever the product $a b$ of two elements of $R$ is contained in $P$, at least one of the elements $a$ and $b$ is contained in $P$. In the case of commutative rings, prime ideals are exactly completely prime ideals. In the case of noncommutative rings, a completely prime ideal is a prime ideal, but a prime ideal is not necessarily a completely prime ideal. Concerning prime ideals, we have the following result for the algebra $H_{r, s}(\Lambda)$.

Corollary 2.1. Suppose the multiplicative group generated by $r$ and $s$ is torsionfree. Then any prime ideal of $H_{r, s}(\Lambda)$ is completely prime.

Proof. The proof follows directly from a result on prime ideals of iterated skew polynomial rings, due to Goodearl and Letzter [2000].

2.4. An algebra isomorphism from $U_{r, s}^{+}(\mathfrak{g})$ onto $\boldsymbol{H}_{r, s}(\Lambda)$. In this subsection, we introduce an algebra isomorphism from the two-parameter quantized enveloping algebra $U_{r, s}^{+}(\mathfrak{g})$ onto the two-parameter Ringel-Hall algebra $H_{r, s}(\Lambda)$. Via this isomorphism, all results established in the previous subsection on $H_{r, s}(\Lambda)$ will be transferred to the two-parameter quantized enveloping algebra $U_{r, s}^{+}(\mathfrak{g})$. For the convenience of this paper, we will awkwardly denote the nontwisted Hall algebra multiplication by $\circ$ in the one-parameter nontwisted generic Ringel-Hall algebra $H_{v}(\Lambda)$ (which can be defined due to the existence of Hall polynomials). Recall also that $v^{2}=q$.

First, we need an important lemma on a two-parameter version of the quantum Serre relations, which was proved to hold in the case of one-parameter nontwisted Ringel-Hall algebra $H_{v}(\Lambda)$.

Lemma 2.4. Let $\alpha_{i} \in \mathscr{P}$ correspond to the simple module $S_{i}$. Then we have the identities

$$
\sum_{k=0}^{1-a_{i j}}(-1)^{k}\left(\begin{array}{c}
1-a_{i j} \\
k
\end{array}\right)_{r_{i} s_{i}^{-1}} c_{i j}^{(k)} u_{\alpha_{i}}^{1-a_{i j}-k} u_{\alpha_{j}} u_{\alpha_{i}}^{k}=0 \quad \text { for } i \neq j
$$

in $H_{r, s}(\Lambda)$, where $c_{i j}^{(k)}=\left(r_{i} s_{i}^{-1}\right)^{k(k-1) / 2} r^{k\langle j, i\rangle} s^{-k\langle i, j\rangle}$ for $i \neq j$.

Proof. The idea of the proof is to reduce these identities to those that have been proved in [Ringel 1990b] to hold for the one-parameter nontwisted generic RingelHall algebra $H_{v}(\Lambda)$. Though this reduction is straightforward, we will provide the details. For convenience, we shall set $m=1-a_{i j}$ in the rest of this proof. 
First, we assume that $i<j$. Then we have $\langle i, j\rangle=d_{i} a_{i j}$ and $\langle j, i\rangle=0$. Therefore,

$$
\begin{aligned}
& \sum_{k=0}^{m}(-1)^{k}\left(\begin{array}{c}
m \\
k
\end{array}\right)_{r_{i} s_{i}^{-1}} c_{i j}^{(k)} u_{\alpha_{i}}^{m-k} u_{\alpha_{j}} u_{\alpha_{i}}^{k} \\
= & \sum_{k=0}^{m}(-1)^{k}\left(\begin{array}{c}
m \\
k
\end{array}\right)_{r_{i} s_{i}^{-1}}\left(r_{i} s_{i}^{-1}\right)^{k(k-1) / 2} r^{k\langle j, i\rangle} s^{-k\langle i, j\rangle} u_{\alpha_{i}}^{m-k} u_{\alpha_{j}} u_{\alpha_{i}}^{k} \\
= & \sum_{k=0}^{m}(-1)^{k}\left(\begin{array}{c}
m \\
k
\end{array}\right)_{r_{i} s_{i}^{-1}}\left(r_{i} s_{i}^{-1}\right)^{k(k-1) / 2} r^{k\langle j, i\rangle} s^{-k\langle i, j\rangle} s^{-(m(m-1) / 2\langle i, i\rangle+(m-k)\langle i, j\rangle+k\langle j, i\rangle)} \\
= & s^{-\left(\langle i, i\rangle m^{2}-m / 2+m\langle i, j\rangle\right)} \sum_{k=0}^{m}(-1)^{k}\left(\begin{array}{c}
m \\
k
\end{array}\right)_{r_{i} s_{i}^{-1}}\left(r_{i} s_{i}^{-1}\right)^{k(k-1) / 2} \circ u_{\alpha_{j}} \circ u_{\alpha_{i}}^{\circ(k)-k)} \circ u_{\alpha_{j}} \circ u_{\alpha_{i}}^{\circ(k)} .
\end{aligned}
$$

Note that the following result was proved for the nontwisted generic Ringel-Hall algebra $H_{v}(\Lambda)$ in [Ringel 1990b]:

$$
\sum_{k=0}^{m}(-1)^{k}\left(\begin{array}{c}
m \\
k
\end{array}\right)_{q^{i}}\left(q^{i}\right)^{k(k-1) / 2} u_{\alpha_{i}}^{\circ(m-k)} \circ u_{\alpha_{j}} \circ u_{\alpha_{i}}^{\circ(k)}=0 .
$$

Due to the existence of Hall polynomials [Ringel 1990a; 1990c], we can set $r s^{-1}=q$. Thus we have proved that the statement is true for $i<j$, as desired.

Now let us assume that $i>j$. Then $\langle i, j\rangle=0$ and $\langle j, i\rangle=d_{j} a_{j i}=d_{i} a_{i j}=$ $d_{i}(1-m)$. Furthermore, we have

$$
\begin{aligned}
& (-1)^{m} \sum_{k=0}^{m}(-1)^{k}\left(\begin{array}{c}
m \\
k
\end{array}\right)_{r_{i} s_{i}^{-1}} c_{i j}^{(k)} u_{\alpha_{i}}^{m-k} u_{\alpha_{j}} u_{\alpha_{i}}^{k} \\
= & \sum_{k=0}^{m}(-1)^{k}\left(\begin{array}{c}
m \\
k
\end{array}\right)_{r_{i} s_{i}^{-1}}\left(r_{i} s_{i}^{-1}\right)^{(m-k)(m-k-1) / 2} r^{(m-k)\langle j, i\rangle} s^{-(m-k)\langle i, j\rangle} u_{\alpha_{i}}^{k} u_{\alpha_{j}} u_{\alpha_{i}}^{m-k} \\
= & \sum_{k=0}^{m}(-1)^{k}\left(\begin{array}{c}
m \\
k
\end{array}\right)_{r_{i} s_{i}^{-1}}\left(r_{i} s_{i}^{-1}\right)^{(m-k)(m-k-1) / 2} r^{(m-k)\langle j, i\rangle} \\
= & r^{\frac{1}{2}\left(m-m^{2}\right)\langle i, i\rangle} \sum_{k=0}^{m}(-1)^{k}\left(\begin{array}{c}
m \\
k
\end{array}\right)_{r_{i} s_{i}^{-1}}\left(r_{i} s_{i}^{-1}\right)^{k(k-1) / 2} u_{\alpha_{i}}^{\circ(k)} \circ u_{\alpha_{j}} \circ u_{\alpha_{i}}^{\circ(m-k)} .
\end{aligned}
$$

The following result was proved for the nontwisted generic Ringel-Hall algebra $H_{v}(\Lambda)$ in [Ringel 1990b]:

$$
\sum_{k=0}^{m}(-1)^{k}\left(\begin{array}{c}
m \\
k
\end{array}\right)_{q^{i}}\left(r^{i}\right)^{k(k-1) / 2} u_{\alpha_{i}}^{\circ(k)} \circ u_{\alpha_{j}} \circ u_{\alpha_{i}}^{\circ(m-k)}=0 .
$$


Once again, thanks to the existence of Hall polynomials, we can set $r s^{-1}=q$ and thus the result follows as desired.

The next result was first proved by Reineke for the case of a finite-dimensional complex simple Lie algebra $\mathfrak{g}$ of type $A, D$ or $E$ in [Reineke 2001], and we show that the result holds for all finite-dimensional complex simple Lie algebras $\mathfrak{g}$. The proof is more or less the same as the one used in [Reineke 2001].

Theorem 2.6 (see also [Reineke 2001]). With the multiplication defined above, $H_{r, s}(\Lambda)$ is an associative $\mathbb{Q}(r, s)$-algebra. In particular, the map

$$
\eta: e_{i} \rightarrow \alpha_{i}
$$

extends to a $\mathbb{Q}(r, s)$-algebra isomorphism

$$
\eta: U_{r, s}^{+}(\mathfrak{g}) \rightarrow H_{r, s}(\Lambda)
$$

Proof. First, note that the quantum Serre relations of $U_{r, s}^{+}(\mathfrak{g})$ are preserved by the map $\eta$. Thus the map $\eta$ indeed defines an algebra homomorphism from the twoparameter quantized enveloping algebra $U_{r, s}^{+}(\mathfrak{g})$ into the two-parameter RingelHall algebra $H_{r, s}(\Lambda)$. It only remains to show that the map $\eta$ is a bijection.

We first show that the map $\eta$ is surjective by verifying that the algebra $H_{r, s}(\Lambda)$ is generated by the elements $u_{i}$ that correspond to the simple modules $S_{i}$ of the algebra $\Lambda$. Let $u_{\alpha}$ be any element in $H_{r, s}(\Lambda)$. Then we have

$$
u_{\alpha}=\left(\prod_{i=1}^{m} \frac{1}{[\alpha(i)]_{\epsilon\left(\boldsymbol{a}_{i}\right)}^{!}}\right) u_{\boldsymbol{a}_{1}}^{\alpha\left(\boldsymbol{a}_{1}\right)} \cdots u_{\boldsymbol{a}_{m}}^{\alpha\left(\boldsymbol{a}_{m}\right)} .
$$

Now it suffices to prove that $u_{\alpha}$ is generated by $u_{i}$ for any $\alpha$ corresponding to an indecomposable module. We prove this claim by using induction. Note that $\zeta(\alpha, \alpha)=0$. Thus, we have

$$
u_{\alpha}=u_{1}^{d_{1}} \cdots u_{n}^{d_{n}}-\sum_{\substack{\beta \neq \alpha \\ \operatorname{dim} \beta=\operatorname{dim} \alpha}} s^{\langle\beta, \beta\rangle} u_{\beta} .
$$

However, one sees that the dimension of the module $u_{\beta}$ is less than that of the module $u_{\alpha}$. Thus by induction on the dimension, we can reduce to the case where $\operatorname{dim}\left(u_{\alpha}\right)=1$. In this case, the only possibility is that $u_{\alpha}=u_{i}$ for some $i$. Thus we have proved the statement that every $u_{\alpha}$ is generated by $u_{i}$, which further implies that the map $\eta$ is a surjective map. We also note that the map $\eta$ is a graded map.

Finally, we show that the map $\eta$ is injective. Let $B:=\mathbb{Q}[r, s]_{(r-1), s-1)}$ denote the localization of the polynomial ring $\mathbb{Q}[r, s]$ at the maximal ideal $(r-1, s-1)$. Then we know that $B=\mathbb{Q}[r, s]_{(r-1, s-1)}$ is a local ring with residue field $\mathbb{Q}$ and fractional field $\mathbb{Q}(r, s)$. Let $U_{B}^{+}$denote the free $B$-algebra generated by the generators $e_{i}$ 
subject to the quantum Serre relations holding in $U_{r, s}^{+}(\mathfrak{g})$. Also let $U_{\mathbb{Q}}^{+}(\mathfrak{g})$ denote the universal enveloping algebra of $\mathfrak{n}^{+}$defined over the base field $\mathbb{Q}$. Then we have

$$
U_{r, s}^{+}(\mathfrak{g})=\mathbb{Q}(r, s) \otimes_{B} U_{B}^{+} \quad \text { and } \quad U_{\mathbb{Q}}^{+}(\mathfrak{g})=\mathbb{Q} \otimes_{B} U_{B}^{+} .
$$

For any $\beta \in \mathbb{N}^{n}$, we have the following result via Nakayama's lemma:

$$
\begin{aligned}
\operatorname{dim}_{\mathbb{Q}} U_{\mathbb{Q}}^{+}(\mathfrak{g})_{\beta} & \left.=\operatorname{dim}_{\mathbb{Q}}\left(\mathbb{Q} \otimes_{B} U_{B}^{+}\right)\right)_{\beta} \\
& \geq \operatorname{dim}_{\mathbb{Q}(r, s)}\left(\mathbb{Q}(r, s) \otimes_{B} U_{B}^{+}\right)_{\beta} \\
& =\operatorname{dim}_{\mathbb{Q}(r, s)} U_{r, s}^{+}(\mathfrak{g})_{\beta} \\
& \geq \operatorname{dim}_{\mathbb{Q}(r, s)} H_{r, s}(\Lambda)_{\beta} .
\end{aligned}
$$

Using [Ringel 1993, Corollary 2] and the PBW-theorem, we also have

$$
\operatorname{dim}_{\mathbb{Q}} U_{\mathbb{Q}}^{+}(\mathfrak{g})_{\beta}=\operatorname{dim}_{\mathbb{Q}(r, s)} H_{r, s}(\Lambda)_{\beta}
$$

Thus we have proved that the map $\eta$ is injective. Therefore, the map $\eta$ is an algebra isomorphism from $U_{r, s}^{+}(\mathfrak{g})$ onto $H_{r, s}(\Lambda)$, as desired.

Based on the previous theorem, the following corollary is in order:

Corollary 2.2. The algebra $U_{r, s}^{+}(\mathfrak{g})$ has a $\mathbb{Q}(r, s)$-basis parameterized by the isomorphism classes of finite-dimensional representations of the algebra $\Lambda$.

Theorem 2.7. All prime ideals of $U_{r, s}^{+}(\mathfrak{g})$ are completely prime under the condition that the multiplicative group generated by $r$ and $s$ is torsion-free.

Proof. This follows since $U_{r, s}(\mathfrak{g})$ is isomorphic to $H_{r, s}(\Lambda)$ as an algebra and since all prime ideals of $H_{r, s}(\Lambda)$ are completely prime under the condition.

\section{The extended two-parameter Ringel-Hall algebras $\overline{H_{r, s}(\Lambda)}$}

In the one-parameter quantum group case, the torus part was added to the RingelHall algebra for the purpose of realizing the Borel subalgebra $U_{q}^{\geq 0}(\mathfrak{g})$ of the oneparameter quantum group $U_{q}(\mathfrak{g})$. In the two-parameter case, we can do the same. Here, we spell out the details. In particular, we will first define the extended Ringel-Hall algebra $\overline{H_{r, s}(\Lambda)}$ by adding the torus part. Then we propose a Hopf algebra structure on this extended two-parameter Ringel-Hall algebra $\overline{H_{r, s}(\Lambda)}$. As an application, we will prove that $U_{r, s}^{\geq 0}(\mathfrak{g})$ is isomorphic to the extended twoparameter Ringel-Hall algebra $\overline{H_{r, s}(\Lambda)}$ as a Hopf algebra. The approach used here is very similar to those in [Ringel 1996; Green 1995; Xiao 1997]. In addition, an analogous result is obtained for the algebra $U_{r, s}^{\leq 0}(\mathfrak{g})$. By patching them together via the triangular decomposition of $U_{r, s}(\mathfrak{g})$, we derive a PBW-basis of $U_{r, s}(\mathfrak{g})$. 
3.1. Extended Ringel-Hall algebras $\overline{H_{r, s}(\Lambda)}$. To realize the Borel subalgebras $U_{r, s}^{\geq 0}(\mathfrak{g})$ and $U_{r, s}^{\leq 0}(\mathfrak{g})$ of the two-parameter quantum group $U_{r, s}(\mathfrak{g})$, we need to enlarge the Ringel-Hall algebras $H_{r, s}(\Lambda)$ defined in the previous section. We are going to enlarge $H_{r, s}(\Lambda)$ by adding the torus part to it. Namely, we will define $\overline{H_{r, s}(\Lambda)}$ to be the free $\mathbb{Q}(r, s)$-module with the basis

$$
\left\{k_{\alpha} u_{\lambda} \mid \alpha \in \mathbb{Z}[I], \lambda \in \mathscr{P}\right\} .
$$

In addition, we are going to define an algebra structure for $\overline{H_{r, s}(\Lambda)}$ by

$$
\begin{array}{rlrl}
u_{\alpha} u_{\beta} & =\sum_{\lambda \in \mathscr{P}} s^{-\langle\alpha, \beta\rangle} F_{u_{\alpha}, u_{\beta}}^{u_{\lambda}}\left(r s^{-1}\right) u_{\lambda} & & \text { for any } \alpha, \beta \in \mathscr{P}, \\
k_{\alpha} u_{\beta}=r^{\langle\beta, \alpha\rangle} s^{-\langle\alpha, \beta\rangle} u_{\beta} k_{\alpha} & & \text { for any } \alpha \in \mathbb{Z}[I], \beta \in \mathscr{P}, \\
k_{\alpha} k_{\beta}=k_{\beta} k_{\alpha} & & \text { for any } \alpha, \beta \in \mathbb{Z}[I] .
\end{array}
$$

Lemma 3.1. For any elements $x, y, z \in \mathbb{Z}[I]$ and $\alpha, \beta, \gamma \in \mathscr{P}$, we have

$$
\left[\left(k_{x} u_{\alpha}\right)\left(k_{y} u_{\beta}\right)\right]\left(k_{x} u_{\alpha}\right)=\left(k_{x} u_{\alpha}\right)\left[\left(k_{y} u_{\beta}\right)\left(k_{z} u_{\gamma}\right)\right] .
$$

In particular, the multiplication defined in $\overline{H_{r, s}(\Lambda)}$ is associative.

Proof. First, we have

$$
\begin{aligned}
& \left(u_{\alpha} u_{\beta}\right) u_{\gamma}=\left(\sum_{\lambda \in \mathscr{P}} s^{-\langle\alpha, \beta\rangle} g_{\alpha \beta}^{\lambda} u_{\lambda}\right) u_{\gamma}=\sum_{\lambda^{\prime} \in \mathscr{P}} s^{-\langle\alpha, \beta\rangle-\langle\alpha+\beta, \gamma\rangle} g_{\alpha \beta \gamma}^{\lambda^{\prime}} u_{\lambda^{\prime}}, \\
& u_{\alpha}\left(u_{\beta} u_{\gamma}\right)=u_{\alpha}\left(\sum_{\lambda \in \mathscr{P}} s^{-\langle\beta, \gamma\rangle} g_{\beta \gamma}^{\lambda} u_{\lambda}\right)=\sum_{\lambda^{\prime} \in \mathscr{P}} s^{-\langle\alpha, \beta+\gamma\rangle-\langle\beta, \gamma\rangle} g_{\alpha \beta \gamma}^{\lambda^{\prime}} u_{\lambda^{\prime}} .
\end{aligned}
$$

So we have just proved that $\left(u_{\alpha} u_{\beta}\right) u_{\gamma}=u_{\alpha}\left(u_{\beta} u_{\gamma}\right)$. In addition, we have the results

$$
\begin{aligned}
& {\left[\left(k_{x} u_{\alpha}\right)\left(k_{y} u_{\beta}\right)\right]\left(k_{z} u_{\gamma}\right)=r^{-\langle\alpha, y\rangle-\langle\alpha+\beta, z\rangle} s^{\langle y, \alpha\rangle+\langle z, \alpha+\beta\rangle} k_{x+y+x} u_{\alpha} u_{\beta} u_{\gamma},} \\
& \left(k_{x} u_{\alpha}\right)\left[\left(k_{y} u_{\beta}\right)\left(k_{z} u_{\gamma}\right)\right]=r^{-\langle\alpha, y+z\rangle-\langle\beta, z\rangle} s^{\langle y+z, \alpha\rangle+\langle z, \beta\rangle} k_{x+y+z} u_{\alpha} u_{\beta} u_{\gamma} .
\end{aligned}
$$

Also, we have $\left(k_{x} u_{\alpha}\right)\left[\left(k_{y} u_{\beta}\right)\left(k_{z} u_{\gamma}\right)\right]=\left[\left(k_{x} u_{\alpha}\right)\left(k_{y} u_{\beta}\right)\right]\left(k_{z} u_{\gamma}\right)$, which further implies that the multiplication is associative.

Proposition 3.1. With the above defined multiplication, $\overline{H_{r, s}(\Lambda)}$ is an associative $\mathbb{Q}(r, s)$-algebra.

Proof. This follows directly from the previous lemma.

Theorem 3.1. The map $\eta$ extends to a $\mathbb{Q}(r, s)$-algebra isomorphism from $U_{r, s}^{\geq 0}(\mathfrak{g})$ onto $\overline{H_{r, s}(\Lambda)}$ via the map $\eta\left(w_{i}\right)=k_{i}$ and $\eta\left(e_{i}\right)=u_{\alpha_{i}}$.

Proof. The proof is straightforward.

Corollary 3.1. The set $\boldsymbol{B}^{+}=\left\{w_{\alpha} \eta^{-1}\left(u_{\lambda}\right) \mid \alpha \in \mathbb{Z}[I], \lambda \in \mathscr{P}\right\}$ is a $\mathbb{Q}(r, s)$-basis of $U_{r, s}^{\geq 0}(\mathfrak{g})$. 
3.2. A Hopf algebra structure on $\overline{\boldsymbol{H}_{r, s}(\boldsymbol{\Lambda})}$. Now we are going to introduce a Hopf algebra structure on the extended two-parameter Ringel-Hall algebra $\overline{H_{r, s}(\Lambda)}$.

Theorem 3.2. The algebra $\overline{H_{r, s}(\Lambda)}$ is a Hopf algebra with the Hopf algebra structure defined as follows.

(1) Multiplication:

$$
\begin{array}{ll}
u_{\alpha} u_{\beta}=\sum_{\lambda \in \mathscr{P}} s^{-\langle\alpha, \beta\rangle} g_{\alpha \beta}^{\lambda} u_{\lambda} & \text { for any } \alpha, \beta \in \mathscr{B}, \\
k_{\alpha} u_{\beta}=r^{\langle\beta, \alpha\rangle} s^{-\langle\alpha, \beta\rangle} u_{\beta} k_{\alpha} & \text { for any } \alpha \in \mathbb{Z}[I], \beta \in \mathscr{P}, \\
k_{\alpha} k_{\beta}=k_{\beta} k_{\alpha} & \text { for any } \alpha, \beta \in \mathbb{Z}[I] .
\end{array}
$$

(2) Comultiplication:

$$
\begin{array}{ll}
\Delta\left(u_{\lambda}\right)=\sum_{\alpha, \beta \in \mathscr{P}} r^{\langle\alpha, \beta\rangle}\left(a_{\alpha} a_{\beta} / a_{\lambda}\right) g_{\alpha \beta}^{\lambda} u_{\alpha} k_{\beta} \otimes u_{\beta} & \text { for any } \lambda \in \mathscr{P}, \\
\Delta\left(k_{\alpha}\right)=k_{\alpha} \otimes k_{\alpha} & \text { for any } \alpha \in \mathbb{Z}[I] .
\end{array}
$$

(3) Counit: $\epsilon\left(u_{\lambda}\right)=0$ for all $\lambda \neq 0$ and $\epsilon\left(k_{\alpha}\right)=1$ for any $\alpha \in \mathscr{P}$.

(4) Antipode:

$$
\begin{aligned}
\sigma\left(u_{\lambda}\right)=\delta_{\lambda, 0}+ & \sum_{m \geq 1}(-1)^{m} \\
& \times \sum_{\pi \in \mathscr{P}, \lambda_{1}, \lambda_{2}, \ldots, \lambda_{m} \in \mathscr{P}_{1}}\left(r s^{-1}\right)^{\sum_{i<j}\left\langle\lambda_{i}, \lambda_{j}\right\rangle} \frac{a_{\lambda_{1}} \cdots a_{\lambda_{m}}}{a_{\lambda}} g_{\lambda_{1} \cdots \lambda_{m}}^{\lambda} g_{\lambda_{1} \cdots \lambda_{m}}^{\pi} k_{-\lambda} u_{\pi}
\end{aligned}
$$

for any element $\lambda \in \mathscr{P}$ and $\sigma\left(k_{\alpha}\right)=k_{-\alpha}$ for any $\alpha \in \mathbb{Z}[I]$.

The proof of this theorem consists of two lemmas.

Lemma 3.2. The comultiplication $\Delta$ is an algebra endomorphism of $\overline{H_{r, s}(\Lambda)}$.

Proof. First, $\Delta\left(k_{x} k_{y}\right)=\Delta\left(k_{x+y}\right)=k_{x+y} \otimes k_{x+y}$. Thus, $\Delta\left(k_{x} k_{y}\right)=\Delta\left(k_{x}\right) \Delta\left(k_{y}\right)$. To prove that $\Delta$ is an algebra homomorphism of $H_{r, s}(\Lambda)$, it suffices to show that $\Delta\left(u_{\alpha} u_{\beta}\right)=\Delta\left(u_{\alpha}\right) \Delta\left(u_{\beta}\right)$. Since

$$
u_{\alpha^{\prime}} u_{\beta^{\prime}}=\sum_{\lambda \in \mathscr{P}} s^{-\left\langle\alpha^{\prime}, \beta^{\prime}\right\rangle} g_{\alpha^{\prime} \beta^{\prime}}^{\lambda} u_{\lambda},
$$

we have

$$
\begin{aligned}
\Delta\left(u_{\alpha^{\prime}} u_{\beta^{\prime}}\right) & =\Delta\left(\sum_{\lambda \in \mathscr{P}} s^{-\langle\alpha, \beta\rangle} g_{\alpha^{\prime} \beta^{\prime}}^{\lambda} u_{\lambda}\right) \\
& =\sum_{\lambda \in \mathscr{P}} s^{-\left\langle\alpha^{\prime}, \beta^{\prime}\right\rangle} g_{\alpha^{\prime} \beta^{\prime}}^{\lambda} \Delta\left(u_{\lambda}\right) \\
& =\sum_{\lambda, \alpha, \beta \in \mathscr{P}} s^{-\left\langle\alpha^{\prime}, \beta^{\prime}\right\rangle} r^{\langle\alpha, \beta\rangle} g_{\alpha^{\prime} \beta^{\prime}}^{\lambda} g_{\alpha \beta}^{\lambda} \frac{a_{\alpha} a_{\beta}}{a_{\lambda}} u_{\alpha} k_{\beta} \otimes u_{\beta}
\end{aligned}
$$


and

$$
\begin{aligned}
& \Delta\left(u_{\alpha^{\prime}}\right) \Delta\left(u_{\beta^{\prime}}\right)=\left(\sum_{\rho, \sigma \in \mathscr{P}} r^{\langle\rho, \sigma\rangle} \frac{a_{\rho} a_{\sigma}}{a_{\alpha^{\prime}}} g_{\rho \sigma}^{\alpha^{\prime}} u_{\rho} k_{\sigma} \otimes u_{\sigma}\right) \\
& \times\left(\sum_{\rho^{\prime}, \sigma^{\prime} \in \mathscr{P}} r^{\left\langle\rho^{\prime}, \sigma^{\prime}\right\rangle} \frac{a_{\rho^{\prime}} a_{a_{\sigma^{\prime}}}}{a_{\beta^{\prime}}} g_{\rho^{\prime} \sigma^{\prime}}^{\beta^{\prime}} u_{\rho^{\prime}} k_{\sigma^{\prime}} \otimes u_{\sigma^{\prime}}\right) \\
& =\sum_{\rho, \sigma, \rho^{\prime}, \sigma^{\prime} \in \mathscr{P}} r^{\langle\rho, \sigma\rangle+\left\langle\rho^{\prime}, \sigma^{\prime}\right\rangle} \frac{a_{\rho} a_{\sigma} a_{\rho^{\prime}} a_{\sigma^{\prime}}}{a_{\alpha^{\prime}} a_{\beta^{\prime}}} \times g_{\rho \sigma}^{\alpha^{\prime}} g_{\rho^{\prime} \sigma^{\prime}}^{\beta^{\prime}} u_{\rho} k_{\sigma} u_{\rho^{\prime}} k_{\sigma^{\prime}} \otimes u_{\sigma} u_{\sigma^{\prime}} \\
& =\sum_{\rho, \sigma, \rho^{\prime}, \sigma^{\prime} \in \mathscr{P}} r^{\langle\rho, \sigma\rangle+\left\langle\rho^{\prime}, \sigma^{\prime}\right\rangle+\left\langle\rho^{\prime}, \sigma\right\rangle} s^{-\left\langle\sigma, \rho^{\prime}\right\rangle-\left\langle\sigma, \sigma^{\prime}\right\rangle-\left\langle\rho, \rho^{\prime}\right\rangle} \frac{a_{\rho} a_{\sigma} a_{\rho^{\prime}} a_{\sigma}}{a_{\alpha^{\prime}} a_{\beta^{\prime}}} \\
& \times g_{\rho \sigma}^{\alpha^{\prime}} g_{\rho^{\prime} \sigma^{\prime}}^{\beta^{\prime}} g_{\rho \rho^{\prime}}^{\alpha} g_{\sigma \sigma^{\prime}}^{\beta} u_{\alpha} k_{\beta} \otimes u_{\beta} \text {. }
\end{aligned}
$$

Note that $\operatorname{dim}\left(u_{\alpha}\right)+\operatorname{dim}\left(u_{\beta}\right)=\operatorname{dim}\left(u_{\lambda}\right)$. Thus, we have

$$
\operatorname{dim}\left(u_{\alpha}\right)+\operatorname{dim}\left(u_{\beta}\right)=\operatorname{dim}\left(u_{\lambda}\right)=\operatorname{dim}\left(u_{\alpha^{\prime}}\right)+\operatorname{dim}\left(u_{\beta^{\prime}}\right)
$$

and

$$
\begin{array}{ll}
\operatorname{dim}\left(u_{\rho}\right)+\operatorname{dim}\left(u_{\sigma}\right)=\operatorname{dim}\left(u_{\alpha^{\prime}}\right), & \operatorname{dim}\left(u_{\rho^{\prime}}\right)+\operatorname{dim}\left(u_{\sigma^{\prime}}\right)=\operatorname{dim}\left(u_{\beta^{\prime}}\right) ; \\
\operatorname{dim}\left(u_{\rho}\right)+\operatorname{dim}\left(u_{\rho^{\prime}}\right)=\operatorname{dim}\left(u_{\alpha}\right), & \operatorname{dim}\left(u_{\sigma}\right)+\operatorname{dim}\left(u_{\sigma^{\prime}}\right)=\operatorname{dim}\left(u_{\beta}\right) .
\end{array}
$$

In addition, we have $k_{\beta}=k_{\sigma} k_{\sigma^{\prime}}$. Thus we have

$$
\begin{aligned}
\langle\alpha, \beta\rangle & =\langle\rho, \sigma\rangle+\left\langle\rho, \sigma^{\prime}\right\rangle+\left\langle\rho^{\prime}, \sigma\right\rangle+\left\langle\sigma, \sigma^{\prime}\right\rangle \\
\left\langle\alpha^{\prime}, \beta^{\prime}\right\rangle & =\left\langle\rho, \rho^{\prime}\right\rangle+\left\langle\rho, \sigma^{\prime}\right\rangle+\left\langle\sigma, \rho^{\prime}\right\rangle+\left\langle\sigma, \sigma^{\prime}\right\rangle .
\end{aligned}
$$

Therefore, we only need to show that

$$
\sum_{\lambda \in \mathscr{P}} g_{\alpha \beta}^{\lambda} g_{\alpha^{\prime} \beta^{\prime}}^{\lambda} \frac{a_{\alpha} a_{\beta} a_{\alpha^{\prime}} a_{\beta^{\prime}}}{a_{\lambda}}=\sum_{\rho, \sigma, \rho^{\prime}, \sigma^{\prime} \in \mathscr{P}}\left(r s^{-1}\right)^{-\left\langle\rho, \sigma^{\prime}\right\rangle} g_{\rho \rho^{\prime}}^{\sigma} g_{\sigma \sigma^{\prime}}^{\beta} g_{\rho \sigma}^{\alpha^{\prime}} g_{\rho^{\prime} \sigma^{\prime}}^{\beta^{\prime}} a_{\rho} a_{\sigma} a_{\rho^{\prime}} a_{\sigma^{\prime}}
$$

but this is true according to Green's formula.

Lemma 3.3. For any $\lambda \in \mathscr{P}$, we have

$$
\mu(\sigma \otimes 1) \Delta\left(u_{\lambda}\right)=\delta_{\lambda 0} \quad \text { and } \quad \mu(1 \otimes \sigma) \Delta\left(u_{\lambda}\right)=\delta_{\lambda 0} .
$$

Proof. First of all, we have

$$
\Delta\left(u_{\lambda}\right)=\sum_{\lambda^{\prime}, \lambda_{m+1} \in \mathscr{P}} \frac{a_{\lambda^{\prime}} a_{\lambda_{m+1}}}{a_{\lambda}} g_{\lambda^{\prime} \lambda_{m+1}}^{\lambda} u_{\lambda^{\prime}} k_{\lambda_{m+1}} \otimes u_{\lambda_{m+1}}
$$

Thus we further have

$$
\mu(\sigma \otimes 1) \Delta\left(u_{\lambda}\right)=\sum_{\lambda^{\prime}, \lambda_{m+1} \in \mathscr{P}} r^{\left\langle\lambda^{\prime}, \lambda_{m+1}\right\rangle} \frac{a_{\lambda^{\prime}} a_{\lambda_{m+1}}}{a_{\lambda}} g_{\lambda^{\prime} \lambda_{m+1}}^{\lambda} k_{-\lambda_{m+1}} \sigma\left(u_{\lambda^{\prime}}\right) u_{\lambda_{m+1}}
$$


To prove the first identity, it suffices to prove that

$$
\sigma\left(u_{\lambda}\right)=\delta_{\lambda 0}-\sum_{\lambda^{\prime} \in \mathscr{P}, \lambda_{m+1} \in \mathscr{P}_{1}} r^{\left\langle\lambda^{\prime}, \lambda_{m+1}\right\rangle} \frac{a_{\lambda^{\prime}} a_{\lambda_{m+1}}}{a_{\lambda}} g_{\lambda^{\prime} \lambda_{m+1}}^{\lambda} k_{-\lambda_{m+1}} \sigma\left(u_{\lambda^{\prime}}\right) u_{\lambda_{m+1}} .
$$

Since we have

$$
\begin{aligned}
\sigma\left(u_{\lambda^{\prime}}\right)=\delta_{\lambda^{\prime} 0}-\sum_{m \geq 1}(-1)^{m} \sum_{\pi^{\prime} \in \mathscr{P}, \lambda_{1}, \ldots, \lambda_{m} \in \mathscr{P}_{1}}\left(r s^{-1}\right)^{\sum_{i<j}\left\langle\lambda_{i}, \lambda_{j}\right\rangle} \frac{a_{\lambda_{1}} \cdots a_{\lambda_{m}}}{a_{\lambda^{\prime}}} \\
\times g_{\lambda_{1} \cdots \lambda_{m}}^{\lambda^{\prime}} g_{\lambda_{1} \cdots \lambda_{m}}^{\pi^{\prime}} k_{-\lambda^{\prime}} u_{\pi^{\prime}},
\end{aligned}
$$

we will have the result

$$
\begin{aligned}
& \sigma\left(u_{\lambda}\right)=\delta_{\lambda 0}-k_{-\lambda} u_{\lambda}-\sum_{\lambda^{\prime}, \lambda_{m+1} \in \mathscr{P}_{1}} r^{\left\langle\lambda^{\prime}, \lambda_{m+1}\right\rangle} \frac{a_{\lambda^{\prime}} a_{\lambda_{m+1}}}{a_{\lambda}} g_{\lambda^{\prime} \lambda_{m+1}}^{\lambda} k_{-\lambda_{m+1}}
\end{aligned}
$$

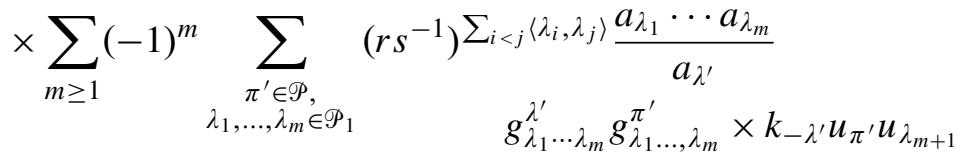

$$
\begin{aligned}
& =\delta_{\lambda 0}-k_{-\lambda} u_{\lambda}-\sum_{m \geq 1}(-1)^{m} \sum_{\substack{\pi^{\prime} \in \mathscr{P} \\
\lambda^{\prime}, \lambda_{1}, \ldots, \lambda_{m} \in \mathscr{P}_{1}}}\left(r s^{-1}\right)^{\sum_{k<j}\left\langle\lambda_{i}, \lambda_{j}\right\rangle} r^{\left\langle\lambda^{\prime}, \lambda_{m+1}\right\rangle} \\
& \times \frac{a_{\lambda_{1} \cdots a_{\lambda_{m}}} a_{\lambda_{m+1}}}{\lambda} g_{\lambda_{1} \cdots \lambda_{m}}^{\lambda^{\prime}} g_{\lambda^{\prime} \lambda}^{\pi^{\prime}} k_{\lambda_{m+1}} k_{-\lambda^{\prime}} \sum_{\pi \in \mathscr{P}} s^{\left\langle\pi^{\prime}, \lambda_{m+1}\right\rangle} g_{\pi^{\prime} \lambda_{m+1}}^{\pi} u_{\pi} \\
& =\delta_{\lambda 0}-\sum_{m \geq 1}(-1)^{m} \sum_{\substack{\pi \in \mathscr{P} \\
\lambda_{1}, \ldots, \lambda_{m} \in \mathscr{P}_{1}}} \frac{a_{\lambda_{1}} \cdots a_{\lambda_{m}} a_{\lambda_{m+1}}}{a_{\lambda}} g_{\lambda_{1} \cdots \lambda_{m} \lambda_{m+1}}^{\lambda} k_{-\lambda} u_{\pi} .
\end{aligned}
$$

Since $g_{\alpha_{1} \cdots \alpha_{i}}^{\lambda} \neq 0$ implies $\operatorname{dim} u_{\lambda}=\operatorname{dim} u_{\alpha_{1}}+\cdots \operatorname{dim} u_{\lambda_{i}}$, we may assume that

$$
\begin{array}{ll}
\operatorname{dim}\left(u_{\lambda^{\prime}}\right)=\operatorname{dim}\left(u_{\lambda_{1}}\right)+\cdots+\operatorname{dim}\left(u_{\lambda_{m}}\right), & \operatorname{dim}\left(u_{\lambda^{\prime}}\right)+\operatorname{dim}\left(u_{\lambda_{m+1}}\right)=\operatorname{dim}\left(u_{\lambda}\right), \\
\operatorname{dim}\left(u_{\pi^{\prime}}\right)=\operatorname{dim}\left(u_{\lambda_{1}}\right)+\cdots+\operatorname{dim}\left(u_{\lambda_{m}}\right), & \operatorname{dim}\left(u_{\pi^{\prime}}\right)+\operatorname{dim}\left(u_{\lambda_{m+1}}\right)=\operatorname{dim}\left(u_{\pi}\right) .
\end{array}
$$

Therefore, we have the result

$$
\begin{aligned}
& \sigma\left(u_{\lambda}\right)= \delta_{\lambda 0}-k_{-\lambda} u_{\lambda}+\sum_{m \geq 2}(-1)^{m} \\
& \sum_{\substack{\pi \in \mathscr{P} \\
\lambda_{1}, \ldots, \lambda_{m} \in \mathscr{P}_{1}}}\left(r s^{-1}\right)^{\sum_{i<j}\left\langle\lambda_{i}, \lambda_{j}\right\rangle} \frac{a_{\lambda_{1}} \cdots a_{\lambda_{m}}}{a_{\lambda}} g_{\lambda_{1} \cdots \lambda_{m}}^{\lambda} g_{\lambda_{1} \cdots \lambda_{m}}^{\pi} k_{-\lambda} u_{\pi} \\
&= \delta_{\lambda 0}+\sum_{m \geq 1}(-1)^{m} \sum_{\substack{\pi \in \mathscr{P} \\
\lambda_{1}, \ldots, \lambda_{m} \in \mathscr{P}_{1}}}\left(r s^{-1}\right)^{\sum_{i<j}\left\langle\lambda_{i}, \lambda_{j}\right\rangle} \frac{a_{\lambda_{1}} \cdots a_{\lambda m}}{a_{\lambda}} \\
& \times g_{\lambda_{1} \cdots \lambda_{m}}^{\lambda} g_{\lambda_{1} \cdots \lambda_{m}}^{\pi} k_{-\lambda} u_{\pi} .
\end{aligned}
$$

So we have proved the statement by the definition of $\sigma$. Similarly, we can verify that $\mu(1 \otimes \sigma) \Delta\left(u_{\lambda}\right)=\delta_{\lambda 0}$. 
Remark 3.1. The proofs of the two lemmas above are slightly modified versions of those in [Xiao 1997].

3.3. A Hopf algebra isomorphism from $U_{r, s}^{\geq 0}(\mathfrak{g})$ onto $\overline{H_{r, s}(\Lambda)}$. Here we prove that the Borel subalgebras $U_{r, s}^{\geq 0}(\mathfrak{g})$ and $U_{r, s}^{\leq 0}(\mathfrak{g})$ of the two-parameter quantum group $U_{r, s}(\mathfrak{g})$ can be realized as the extended two-parameter Ringel-Hall algebras $\overline{H_{r, s}(\Lambda)}$ and $\overline{H_{s^{-1}, r^{-1}}(\Lambda)}$ as Hopf algebras. As a result, we shall derive a PBWbasis for the algebra $U_{r, s}(\mathfrak{g})$.

Theorem 3.3. We have

$$
U_{r, s}^{\geq 0}(\mathfrak{g}) \cong \overline{H_{r, s}(\Lambda)} \quad \text { and } \quad U_{r, s}^{\leq 0}(\mathfrak{g}) \cong \overline{H_{s^{-1}, r^{-1}}(\Lambda)}
$$

as Hopf algebras.

Proof. Let us define a map $\phi: U_{r, s}^{\geq 0}(\mathfrak{g}) \rightarrow \overline{H_{r, s}(\Lambda)}$ by setting $\phi\left(E_{i}\right)=u_{S_{i}}$ and $\phi\left(w_{i}\right)=k_{i}$. Then it is easy to verify that $\phi$ is a bijection and respects the Hopf algebra structures. Thus it is a Hopf algebra isomorphism. Similarly, we can prove that $U_{r, s}^{\leq 0}(\mathfrak{g})$ is isomorphic to $\overline{H_{s^{-1}, r^{-1}}(\Lambda)}$ as a Hopf algebra.

Let $\boldsymbol{B}^{-}$be the basis constructed for $U_{r, s}^{\leq 0}(\mathfrak{g})$ via the algebra $\overline{H_{s^{-1}, r^{-1}}(\Lambda)}$; then we have the following:

Corollary 3.2. The set $\boldsymbol{B}^{+} \cup \boldsymbol{B}^{-}$is a $\mathbb{Q}(r, s)$-basis for the two-parameter quantum groups $U_{r, s}(\mathfrak{g})$.

\section{Acknowledgment}

I would like to thank the referee for various comments and suggestions, which have dramatically helped to improve the presentation of the material in this paper.

\section{References}

[Artin et al. 1991] M. Artin, W. Schelter, and J. Tate, "Quantum deformations of GLn", Comm. Pure Appl. Math. 44:8-9 (1991), 879-895. MR 92i:17014 Zbl 0753.17015

[Benkart and Witherspoon 2004a] G. Benkart and S. Witherspoon, "Restricted two-parameter quantum groups", pp. 293-318 in Representations of finite dimensional algebras and related topics in Lie theory and geometry (Toronto, 2002), edited by V. Dlab and C. M. Ringel, Fields Inst. Commun. 40, Amer. Math. Soc., Providence, RI, 2004. MR 2005b:17027 Zbl 1048.16020

[Benkart and Witherspoon 2004b] G. Benkart and S. Witherspoon, “Two-parameter quantum groups and Drinfel'd doubles", Algebr. Represent. Theory 7:3 (2004), 261-286. MR 2005g:17028 Zbl 1113.16041

[Benkart et al. 2006] G. Benkart, S.-J. Kang, and K.-H. Lee, "On the centre of two-parameter quantum groups", Proc. Roy. Soc. Edinburgh Sect. A 136:3 (2006), 445-472. MR 2007a:17019 Zbl 1106.17013

[Bergeron et al. 2006] N. Bergeron, Y. Gao, and N. Hu, "Drinfel'd doubles and Lusztig's symmetries of two-parameter quantum groups", J. Algebra 301:1 (2006), 378-405. MR 2007e:17010 Zbl 1148.17007 
[Bernšteĭn et al. 1973] I. N. Bernšteйn, I. M. Gel'fand, and V. A. Ponomarev, "Coxeter functors, and Gabriel's theorem", Uspehi Mat. Nauk 28:2(170) (1973), 19-33. In Russian. MR 52 \#13876 Zbl 0269.08001

[Chin and Musson 1996] W. Chin and I. M. Musson, "Multiparameter quantum enveloping algebras”, J. Pure Appl. Algebra 107:2-3 (1996), 171-191. MR 97c:17016 Zbl 0859.17004

[Dlab and Ringel 1975] V. Dlab and C. M. Ringel, "On algebras of finite representation type", $J$. Algebra 33 (1975), 306-394. MR 50 \#9974 Zbl 0332.16014

[Dlab and Ringel 1976] V. Dlab and C. M. Ringel, Indecomposable representations of graphs and algebras, Mem. Amer. Math. Soc. 173, American Mathematical Society, Providence, RI, 1976. MR 56 \#5657 Zbl 0332.16015

[Dobrev and Parashar 1993] V. K. Dobrev and P. Parashar, "Duality for multiparametric quantum GL(n)”, J. Phys. A 26:23 (1993), 6991-7002. MR 95d:81053 Zbl 0821.17009

[Doi and Takeuchi 1994] Y. Doi and M. Takeuchi, "Multiplication alteration by two-cocycles — the quantum version”, Comm. Algebra 22:14 (1994), 5715-5732. MR 95j:16043 Zbl 0821.16038

[Gabriel 1972] P. Gabriel, "Unzerlegbare Darstellungen, I", Manuscripta Math. 6 (1972), 71-103. Corrected in 6 (1972), 309. MR 48 \#11212 Zbl 0232.08001

[Gabriel 1973] P. Gabriel, "Indecomposable representations, II", pp. 81-104 in Convegno di Algebra Commutativa, INDAM (Rome, 1971), Symposia Mathematica 11, Academic Press, London, 1973. MR 49 \#5132 Zbl 0276.16001

[Goodearl and Letzter 2000] K. R. Goodearl and E. S. Letzter, "The Dixmier-Moeglin equivalence in quantum coordinate rings and quantized Weyl algebras", Trans. Amer. Math. Soc. 352:3 (2000), 1381-1403. MR 2000j:16040 Zbl 0978.16040

[Green 1995] J. A. Green, "Hall algebras, hereditary algebras and quantum groups", Invent. Math. 120:2 (1995), 361-377. MR 96c:16016 Zbl 0836.16021

[Hu and Pei 2008] N. Hu and Y. Pei, "Notes on 2-parameter quantum groups, I", Sci. China Ser. A 51:6 (2008), 1101-1110. MR 2009c:17016 Zbl 1145.81381

[Hu et al. 2008] N. Hu, M. Rosso, and H. Zhang, "Two-parameter quantum affine algebra $U_{r, s}\left(\widehat{\mathfrak{s l}}_{n}\right)$, Drinfel'd realization and quantum affine Lyndon basis", Comm. Math. Phys. 278:2 (2008), 453486. MR 2009b:17033

[Jing 1992] N. H. Jing, "Quantum groups with two parameters", pp. 129-138 in Deformation theory and quantum groups with applications to mathematical physics (Amherst, MA, 1990), edited by M. Gerstenhaber and J. Stasheff, Contemp. Math. 134, Amer. Math. Soc., Providence, RI, 1992. MR 94b:17022 Zbl 0774.17015

[Kharchenko 1999] V. K. Kharchenko, "A quantum analogue of the Poincaré-Birkhoff-Witt theorem”, Algebra Log. 38:4 (1999), 476-507, 509. In Russian; translated in Algebra and Logic 38:4 (1999), 259-276. MR 2001f:16075

[Kharchenko 2002] V. K. Kharchenko, "A combinatorial approach to the quantification of Lie algebras”, Pacific J. Math. 203:1 (2002), 191-233. MR 2003b:17018 Zbl 1069.17008

[Kulish 1990] P. P. Kulish, "A two-parameter quantum group and a gauge transformation", Zap. Nauchn. Sem. Leningrad. Otdel. Mat. Inst. Steklov. (LOMI) 180:Voprosy Kvant. Teor. Polya i Statist. Fiz. 9 (1990), 89-93, 180. In Russian; translated in J. Math. Sci., New York 68:2 (1994), 220-222. MR 91j:81029 Zbl 0792.17011

[Lusztig 1990] G. Lusztig, "Canonical bases arising from quantized enveloping algebras", J. Amer. Math. Soc. 3:2 (1990), 447-498. MR 90m:17023 Zbl 0703.17008

[Lusztig 1993] G. Lusztig, Introduction to quantum groups, Progress in Mathematics 110, Birkhäuser, Boston, MA, 1993. MR 94m:17016 Zbl 0788.17010 
[Reineke 2001] M. Reineke, "Generic extensions and multiplicative bases of quantum groups at $q=0$ ”, Represent. Theory 5 (2001), 147-163. MR 2002c:17029 Zbl 1050.17015

[Reshetikhin 1990] N. Reshetikhin, "Multiparameter quantum groups and twisted quasitriangular Hopf algebras", Lett. Math. Phys. 20:4 (1990), 331-335. MR 91k:17012 Zbl 0719.17006

[Ringel 1976] C. M. Ringel, "Representations of $K$-species and bimodules", J. Algebra 41:2 (1976), 269-302. MR 54 \#10340 Zbl 0338.16011

[Ringel 1990a] C. M. Ringel, "Hall algebras", pp. 433-447 in Topics in algebra (Warsaw, 1988), vol. 1, edited by S. Balcerzyk et al., Banach Center Publ. 26, PWN, Warsaw, 1990. MR 93f:16027 Zbl 0778.16004

[Ringel 1990b] C. M. Ringel, "Hall algebras and quantum groups", Invent. Math. 101:3 (1990), 583-591. MR 91i:16024 Zbl 0735.16009

[Ringel 1990c] C. M. Ringel, "Hall polynomials for the representation-finite hereditary algebras", Adv. Math. 84:2 (1990), 137-178. MR 92e:16010 Zbl 0799.16013

[Ringel 1993] C. M. Ringel, "Hall algebras revisited", pp. 171-176 in Quantum deformations of algebras and their representations (Rehovot, 1991/1992), edited by A. Joseph and S. Shnider, Israel Math. Conf. Proc. 7, Bar-Ilan Univ., Ramat Gan, 1993. MR 94k:16021 Zbl 0852.17009

[Ringel 1996] C. M. Ringel, "PBW-bases of quantum groups", J. Reine Angew. Math. 470 (1996), 51-88. MR 97d:17009 Zbl 0840.17010

[Sudbery 1990] A. Sudbery, "Consistent multiparameter quantisation of GL(n)", J. Phys. A 23:15 (1990), L697-L704. MR 91m:17022 Zbl 0722.17007

[Takeuchi 1990] M. Takeuchi, "A two-parameter quantization of GL(n) (summary)", Proc. Japan Acad. Ser. A Math. Sci. 66:5 (1990), 112-114. MR 92f:16049

[Xiao 1997] J. Xiao, "Drinfeld double and Ringel-Green theory of Hall algebras", J. Algebra 190:1 (1997), 100-144. MR 98a:16018 Zbl 0874.16026

Received June 23, 2009. Revised January 5, 2010.

XIN TANG

Department of Mathematics \& Computer Science

FAYETTEVILLE STATE UNIVERSITY

1200 MURCHISON ROAD

FAYETTEVILLE, NC 28301

xtang@uncfsu.edu 


\title{
PACIFIC JOURNAL OF MATHEMATICS
}

\author{
http://www.pjmath.org \\ Founded in 1951 by \\ E. F. Beckenbach (1906-1982) and F. Wolf (1904-1989)
}

\section{EDITORS}

V. S. Varadarajan (Managing Editor)

Department of Mathematics

University of California

Los Angeles, CA 90095-1555

pacific@math.ucla.edu

Vyjayanthi Chari

Department of Mathematics

University of California

Riverside, CA 92521-0135

chari@math.ucr.edu

Robert Finn

Department of Mathematics Stanford University

Stanford, CA 94305-2125

finn@math.stanford.edu

Kefeng Liu

Department of Mathematics

University of California

Los Angeles, CA 90095-1555

liu@math.ucla.edu
Darren Long

Department of Mathematics

University of California

Santa Barbara, CA 93106-3080

long@math.ucsb.edu

Jiang-Hua Lu

Department of Mathematics

The University of Hong Kong

Pokfulam Rd., Hong Kong jhlu@maths.hku.hk

Alexander Merkurjev

Department of Mathematics

University of California

Los Angeles, CA 90095-1555

merkurev@math.ucla.edu
Sorin Popa

Department of Mathematics University of California

Los Angeles, CA 90095-1555 popa@math.ucla.edu

Jie Qing

Department of Mathematics

University of California

Santa Cruz, CA 95064

qing@cats.ucsc.edu

Jonathan Rogawski

Department of Mathematics

University of California

Los Angeles, CA 90095-1555

jonr@math.ucla.edu

\section{PRODUCTION}

pacific@math.berkeley.edu

\begin{abstract}
Silvio Levy, Scientific Editor Matthew Cargo, Senior Production Editor
\end{abstract}
ACADEMIA SINICA, TAIPEI

CALIFORNIA INST. OF TECHNOLOGY

INST. DE MATEMÁTICA PURA E APLICADA

KEIO UNIVERSITY

MATH. SCIENCES RESEARCH INSTITUTE

NEW MEXICO STATE UNIV.

OREGON STATE UNIV.

\section{SUPPORTING INSTITUTIONS}

STANFORD UNIVERSITY
UNIV. OF BRITISH COLUMBIA
UNIV. OF CALIFORNIA, BERKELEY
UNIV. OF CALIFORNIA, DAVIS
UNIV. OF CALIFORNIA, LOS ANGELES
UNIV. OF CALIFORNIA, RIVERSIDE
UNIV. OF CALIFORNIA, SAN DIEGO
UNIV. OF CALIF., SANTA BARBARA

UNIV. OF CALIF., SANTA CRUZ

UNIV. OF MONTANA

UNIV. OF OREGON

UNIV. OF SOUTHERN CALIFORNIA

UNIV. OF UTAH

UNIV. OF WASHINGTON

WASHINGTON STATE UNIVERSITY

These supporting institutions contribute to the cost of publication of this Journal, but they are not owners or publishers and have no responsibility for its contents or policies.

See inside back cover or www.pjmath.org for submission instructions.

The subscription price for 2010 is US \$420/year for the electronic version, and \$485/year for print and electronic.

Subscriptions, requests for back issues from the last three years and changes of subscribers address should be sent to Pacific Journal of Mathematics, P.O. Box 4163, Berkeley, CA 94704-0163, U.S.A. Prior back issues are obtainable from Periodicals Service Company, 11 Main Street, Germantown, NY 12526-5635. The Pacific Journal of Mathematics is indexed by Mathematical Reviews, Zentralblatt MATH, PASCAL CNRS Index, Referativnyi Zhurnal, Current Mathematical Publications and the Science Citation Index.

The Pacific Journal of Mathematics (ISSN 0030-8730) at the University of California, c/o Department of Mathematics, 969 Evans Hall, Berkeley, CA 94720-3840, is published monthly except July and August. Periodical rate postage paid at Berkeley, CA 94704, and additional mailing offices. POSTMASTER: send address changes to Pacific Journal of Mathematics, P.O. Box 4163, Berkeley, CA 94704-0163.

PJM peer review and production are managed by EditFLOW ${ }^{\mathrm{TM}}$ from Mathematical Sciences Publishers.

PUBLISHED BY PACIFIC JOURNAL OF MATHEMATICS

at the University of California, Berkeley 94720-3840

A NON-PROFIT CORPORATION

Typeset in LATEX

Copyright $(\mathrm{C} 2010$ by Pacific Journal of Mathematics 


\section{PACIFIC JOURNAL OF MATHEMATICS}

Volume $247 \quad$ No. $1 \quad$ September 2010

Classification results for easy quantum groups

TEOdor BANiCA, StePhen CURRAN and Roland SPEICHER

Batalin-Vilkovisky coalgebra of string topology

XiAOJUn CHEN and WeE LiAnG GAN

Invariant Finsler metrics on polar homogeneous spaces

SHAOQIANG DENG

A proof of the Concus-Finn conjecture

KIRK E. LANCASTER

The existence and monotonicity of a three-dimensional transonic shock in a finite nozzle with axisymmetric exit pressure

JUn Li, ZHOUPING XIN and HUICHENG YiN

Bi-Hamiltonian flows and their realizations as curves in real semisimple homogeneous manifolds

GLORIA MARÍ BEFFA

Closed orbits of a charge in a weakly exact magnetic field

WILL J. MERRY

Ringel-Hall algebras and two-parameter quantized enveloping algebras

XIN TANG

A new probability distribution with applications

MINGJIN WANG 